\title{
Stability of Polygenic Scores Across Discovery Genome-Wide Association Studies
}

Laura M. Schultz, ${ }^{1,}{ }^{*}$ Alison K. Merikangas, ${ }^{1,2,3}$ Kosha Ruparel, ${ }^{2,4}$ Sébastien Jacquemont, ${ }^{5}$ David C. Glahn, ${ }^{6,7}$ Raquel E. Gur, ${ }^{2,4,8}$ Ran Barzilay, ${ }^{2,4,8}$ Laura Almasy ${ }^{1,2,3}$

${ }^{1}$ Department of Biomedical and Health Informatics, Children's Hospital of Philadelphia, Philadelphia, PA, 19104, USA

${ }^{2}$ Lifespan Brain Institute, Children's Hospital of Philadelphia and Penn Medicine, Philadelphia, PA, 19104, USA

${ }^{3}$ Department of Genetics, Perelman School of Medicine, University of Pennsylvania, Philadelphia, PA, 19104, USA

${ }^{4}$ Department of Psychiatry, Perelman School of Medicine, University of Pennsylvania, Philadelphia, PA, 19104, USA

${ }^{5}$ Sainte Justine Research Center, University of Montréal, Montréal, QC, H3T 1C5, Canada

${ }^{6}$ Tommy Fuss Center for Neuropsychiatric Disease Research, Boston Children's Hospital, Boston, MA, USA

${ }^{7}$ Department of Psychiatry, Harvard Medical School, Boston, MA, USA

${ }^{8}$ Department of Child Adolescent Psychiatry and Behavioral Sciences, Children's Hospital of Philadelphia, Philadelphia, PA, 19104, USA

* Correspondence: schultzl1@chop.edu 


\section{Abstract}

Polygenic scores (PGS) are commonly evaluated in terms of their predictive accuracy at the population level by the proportion of phenotypic variance they explain. To be useful for precision medicine applications, they also need to be evaluated at the individual patient level when phenotypes are not necessarily already known. Hence, we investigated the stability of PGS in European-American (EUR)- and African-American (AFR)-ancestry individuals from the Philadelphia Neurodevelopmental Cohort (PNC) and the Adolescent Brain Cognitive Development $(A B C D)$ cohort using different discovery GWAS for post-traumatic stress disorder (PTSD), type-2 diabetes (T2D), and height. We found that pairs of EUR-ancestry GWAS for the same trait had genetic correlations $>0.92$. However, PGS calculated from pairs of sameancestry and different-ancestry GWAS had correlations that ranged from $<0.01$ to 0.74 . PGS stability was higher for GWAS that explained more of the trait variance, with height PGS being more stable than PTSD or T2D PGS. Focusing on the upper end of the PGS distribution, different discovery GWAS do not consistently identify the same individuals in the upper quantiles, with the best case being $60 \%$ of individuals above the $80^{\text {th }}$ percentile of PGS overlapping from one height GWAS to another. The degree of overlap decreases sharply as higher quantiles, less heritable traits, and different-ancestry GWAS are considered. PGS computed from different discovery GWAS have only modest correlation at the level of the individual patient, underscoring the need to proceed cautiously with integrating PGS into precision medicine applications. 


\section{Introduction}

Polygenic scores (PGS) are increasingly being used to draw inferences regarding genetic contributions to a variety of complex anthropometric and disease-related traits. Numerous methods have been developed for computing PGS for a target population using summary statistics from a discovery genome-wide association study (GWAS) run for an independent population, with newer Bayesian-based techniques such as LDpred, ${ }^{1}$ SBayesR, ${ }^{2}$ and $\mathrm{PRS}-\mathrm{CS}^{3}$ generally yielding more predictive PGS than those produced using older methodologies that rely on a combination of linkage disequilibrium (LD) clumping and $P$-value thresholding. ${ }^{4}$

One goal is to utilize PGS in clinical settings to facilitate the diagnosis and treatment of a wide range of heritable diseases, ${ }^{5}$ such as inflammatory bowel disease, ${ }^{6}$ diabetes, ${ }^{7}$ cardiovascular disease, ${ }^{8 ;} 9$ cancer, ${ }^{10}$ Alzheimer's disease, ${ }^{11}$ attention-deficit/hyperactivity disorder, ${ }^{12}$ major depressive disorder, ${ }^{13}$ bipolar disorder, ${ }^{14}$ and schizophrenia. ${ }^{15}$ While progress has been made towards reaching this goal, ${ }^{16-19}$ numerous challenges remain to be solved. $5 ;$ 20-23 Given that the GWAS required for computing PGS have been disproportionately run for European-ancestry populations, ${ }^{24-28}$ a fundamental challenge will be ensuring that diverse populations have equitable access to medically beneficial PGS, ${ }^{29}$ as it has been demonstrated that that PGS are less predictive when the target and discovery populations have differing genetic ancestry or varying degrees of admixture. ${ }^{30-34}$

Previous studies have evaluated PGS performance in terms of how well they predict phenotypes at the population level. However, if PGS are going to be adopted in the precisionmedicine setting, it is also necessary to examine how well PGS perform at predicting the risk for 
individual patients. ${ }^{35}$ To this end, we examined the stability of PGS computed for individuals across discovery GWAS. Specifically, we evaluated the correlations between the PGS computed for EUR and AFR individuals from pairs of same- and different-ancestry discovery GWAS for post-traumatic stress disorder, ${ }^{36 ; 37}$ type 2 diabetes, ${ }^{38-40}$ and height. ${ }^{41 ; 42}$ These specific traits were chosen because they had sufficiently powered, publicly available AFR-ancestry GWAS. We also addressed the question of whether the same individuals were consistently identified as belonging to the top PGS quantiles. For this work, we targeted European-American (EUR) and African-American (AFR) youth from the Philadelphia Neurodevelopmental Cohort (PNC) and the Adolescent Brain Cognitive Development Study (ABCD).

\section{Subjects and Methods}

\section{Philadelphia Neurodevelopmental Cohort (PNC)}

Genotype data for the PNC, a population-based sample of youth who were ages 8-21 at the time of study enrollment, ${ }^{43}$ were obtained from dbGaP (phs000607.v2.p2). Biological samples from PNC subjects were genotyped in fifteen batches (Table S1) using ten different types of Affymetrix and Illumina arrays by the Center for Applied Genomics at the Children's Hospital of Philadelphia. ${ }^{44}$ Analysis was limited to the 5,239 EUR and 3,260 AFR ancestry individuals for whom genotype data were available after the quality-control (QC) process described below.

\section{Adolescent Brain Cognitive Development Study (ABCD)}

Results were replicated using post-QC genotype data for 5,815 EUR and 1,741 AFR individuals in the independent $A B C D$ cohort (NDA \#2573, fix release 2.0.1). This cohort is 
comprised of adolescents who were ages 9-10 at the time that their saliva samples were collected for genotyping. ${ }^{45}$ The Rutgers University Cell and DNA Repository stored and genotyped all samples using the Affymetrix NIDA SmokeScreen array.

\section{Quality Control and Imputation}

The PNC dataset was processed by array batch and merged after imputation, whereas the $A B C D$ dataset was processed as a single batch. For each batch, PLINK $1.9^{46}$ was used to remove single nucleotide polymorphisms (SNPs) with $>5 \%$ missingness, samples with more than $10 \%$ missingness, and samples with a genotyped sex that did not match the reported sex phenotype. As a final step, each batch was checked with a pre-imputation perl script that compared SNP frequencies against the 1000 Genomes ALL reference panel. ${ }^{47}$ This script fixed strand reversals and improper Ref/Alt assignments and also removed palindromic $A / T$ and $C / G$ SNPs with minor allele frequency $(M A F)>0.4$, SNPs with alleles that did not match the reference panel, SNPs with allele frequencies differing by more than 0.2 from the reference, and SNPs not present in the reference panel.

Genotypes were phased (Eagle v.2.4) and imputed by chromosome to the 1000 Genomes Other/Mixed GRCh37/hg19 reference panel (Phase 3 v.5) using Minimac 4 via the Michigan Imputation Server. ${ }^{48}$ The fifteen imputation batches for the PNC dataset were merged by chromosome, and then post-imputation QC was run on the merged chromosome files using bcftools. ${ }^{49}$ Only polymorphic sites with imputation quality $R^{2} \geq 0.7$ and MAF $\geq 0.01$ were included in the final PLINK 1.9 hard-call PNC and ABCD post-imputation datasets. 


\section{Ancestry and Kinship Analysis}

Multi-dimensional scaling (MDS) was conducted using KING (v.2.2.4) $)^{50}$ to identify the top ten ancestry principal components (PCs) for each sample. These PCs were projected onto the 1000 Genomes PC space, and genetic ancestry was inferred using the $1071^{51}$ support vector machines package in $\mathrm{R}^{52}$ (Figure 1). Based on these inferences, AFR- and EUR-ancestry cohorts were created for the PNC and ABCD datasets; all other ancestry groups were excluded from further analysis. A second round of unprojected MDS was then performed within the EUR- and AFR-ancestry groups to produce ten PCs that were regressed out of the standardized PGS to adjust for array batch effects and genetic ancestry (Figures S1-S5).

KING was also used to identify all pairwise relationships out to third degree relatives based on estimated kinship coefficients and inferred IBD segments. Although the PNC was not recruited as a family study, it does include some related individuals (i.e., siblings and cousins). We ran a sensitivity analysis using a reduced PNC dataset that included only one individual from each family (chosen as the lowest individual ID number for a given family ID number), which reduced the size of the PNC EUR cohort from 5,239 to 4,928 and the AFR cohort from 3,260 to 2,954. After establishing that the PNC PTSD PGS correlation results obtained using only unrelated individuals did not differ meaningfully from those obtained using the full dataset (Tables S4 and S5), we performed all subsequent analyses using the complete EUR and AFR cohorts.

\section{Polygenic Score Computation with PRS-CS}

PRS-CS 3 was used to infer posterior effects by chromosome for the SNPs in a given dataset that overlapped with both the discovery GWAS summary statistics and an external 1000 
Genomes LD panel that was matched to the ancestry group used for the discovery GWAS.

Posterior effects were only inferred for SNPs located on the 22 autosomal chromosomes. PGS

for the EUR and AFR subsets of PNC and ABCD were computed using both EUR and AFR

discovery GWAS for post-traumatic stress disorder (PTSD), ${ }^{36 ;} 37$ Type-2 diabetes (T2D), ${ }^{38-40}$ and

height ${ }^{41 ; 42}$ (Table 1). To ensure convergence of the underlying Gibbs sampler algorithm, we ran

25,000 Markov chain Monte Carlo (MCMC) iterations and designated the first 10,000 MCMC

iterations as burn-in. The PRS-CS global shrinkage parameter was set to 0.01 when the

discovery GWAS had a SNP sample size that was less than 200,000; otherwise, it was learned

from the data using a fully Bayesian approach. Default settings were used for all other PRS-CS

parameters. Given the stochastic nature of the Bayesian algorithm used by PRS-CS, PGS

replicability was confirmed by completing multiple PRS-CS runs using the same discovery

GWAS. Raw PGS were produced from the posterior effects using PLINK 1.9. $\mathrm{R}^{52}$ was used to standardize the PGS for a given cohort to mean $=0$ and $S D=1$. Standardized PGS were then adjusted by regressing out the first ten within-ancestry PCs.

\section{SNP Heritability Estimation with LDSC}

LD score regression (LDSC) ${ }^{53 ; 54}$ was used to calculate SNP-based heritability $\left(h_{S N P}^{2}\right)$ on the observed scale for each EUR-ancestry discovery GWAS that we used to generate PGS. We also calculated $h_{S N P}^{2}$ on the liability scale for PTSD and T2D by incorporating the sample prevalence and estimated population prevalence into the calculation (Table S14). The standard error associated with a given $h_{S N P}^{2}$ calculation was estimated using a block jackknife over blocks of adjacent SNPs. Given that LDSC may yield biased estimates for admixed populations ${ }^{55}$ and 
that we only had access to GWAS summary statistics, we did not calculate SNP heritability for the AFR-ancestry discovery GWAS.

\section{Statistical Analysis}

All statistics and graphical displays were generated using R. ${ }^{52}$ Pearson correlation coefficients were calculated to assess the strength of correlations between PC-adjusted standardized PGS that were calculated for a given trait using different discovery GWAS. Statistical significance was determined with two-tailed $t$-tests for linear association. For each comparison, counts were also made of the number of samples in common at or above the $80^{\text {th }}$ percentile, the $90^{\text {th }}$ percentile, and the $95^{\text {th }}$ percentile of the adjusted standardized PGS distributions.

\section{Results}

\section{Reproducibility of Bayesian Posterior Effects}

Given that PRS-CS relies on Bayesian methodology to infer posterior effects for the SNPS on each chromosome ${ }^{3}$ it was necessary to confirm that we had used enough Markov chain Monte Carlo (MCMC) iterations and burn-in trials to ensure convergence of the underlying Gibbs sampler algorithm. We checked for convergence indirectly by assessing the correlation between the posterior effects calculated across multiple runs for a given chromosome (Figure 2). The PRS-CS default setting of $1000 \mathrm{MCMC}$ iterations with the first 500 iterations serving as burn-in produced relatively inconsistent posterior effects $(r \approx 0.8)$, suggesting incomplete convergence. The correlation between the posterior effects computed during multiple runs of PRS-CS improved to $r \approx 0.98$ when we increased the number of MCMC iterations to 10,000 
(5,000 burn-in) and further improved to $r>0.99$ for both large and small chromosomes when we used 25,000 MCMC iterations (10,000 burn-in). Given that the computational time increases substantially as more MCMC iterations are run, we opted to use 25,000 MCMC iterations with the first 10,000 as burn-in rather than pursuing even stronger correlations.

\section{Reproducibility of PGS Computed from the Same Discovery GWAS}

The next concern was whether the PGS calculated by PLINK 1.9 from the Bayesian posterior effects would also be reproducible across PRS-CS runs. To address this question, we ran PRS-CS twice using the Psychiatric Genomics Consortium (PGC) Freeze 2 PTSD discovery GWAS $^{37}$, and calculated PGS from both sets of posterior effects. For both the EUR and AFR PNC cohorts, the correlation between the adjusted PGS was greater than 0.999 (Figure 3). Hence, we are confident that PRS-CS yields reproducible PGS for a given discovery GWAS provided that enough MCMC iterations are used.

\section{Stability of PGS Computed from Different Same-Ancestry Discovery GWAS}

Of the three traits that we analyzed, only PTSD had two publicly available AFR-ancestry GWAS. ${ }^{36 ;} 37$ We computed PGS using both GWAS for each AFR-ancestry individual and then assessed the correlation between the two sets of PGS (Figure 4). We found a moderately strong positive correlation between the PGS computed from the PGC Freeze $1^{36}$ and Freeze $2^{37}$ AFR-ancestry PTSD GWAS for the AFR-ancestry cohorts of both PNC $(r=0.696, t(3258)=55.26$, $\left.P<2 \times 10^{-16}\right)$ and $\operatorname{ABCD}\left(r=0.657, t(1739)=36.34, P<2 \times 10^{-16}\right)$.

The wider availability of EUR-ancestry GWAS allowed us to compute PGS for EURancestry individuals using pairs of EUR-ancestry discovery GWAS for PTSD, ${ }^{36 ; 37}{ }^{T 2 D},{ }^{38 ; 39}$ and height $^{41 ; 42}$ (Figure 5). Statistically significant positive correlations between the pairs of PGS 
were observed for all three traits for both the PNC (Table S8) and ABCD (Table S9) EUR-ancestry cohorts, with the strongest association observed between the height PGS (PNC: $r=0.736$;

ABCD: $r=0.734$ ) and the weakest observed for the PTSD PGS (PNC: $r=0.392 ; A B C D: r=0.378$ ).

\section{Stability of PGS Computed from Different-Ancestry Discovery GWAS}

Given the scarcity of AFR-ancestry GWAS, it is often tempting to compute PGS for AFRancestry individuals using EUR-ancestry discovery GWAS. To assess the feasibility of this approach, we computed PGS for AFR-ancestry individuals in PNC and ABCD using both AFRancestry discovery GWAS and EUR-ancestry GWAS and then assessed the correlation between the two sets of PGS (Figure 6).

For PTSD, there was no significant correlation between the PGS computed from the newer Freeze 2 PGC AFR and EUR discovery GWAS $^{37}$ for AFR-ancestry individuals in either PNC $(r=0.00356, t(3258)=0.203, P=0.839)$ or $\operatorname{ABCD}(r=0.00283, t(1739)=0.118, P=0.906)$. The AFR PGS computed using the Freeze 1 PGC PTSD AFR and EUR discovery GWAS ${ }^{36}$ were uncorrelated for $\mathrm{ABCD}(r=-0.00320, t(1739)=-0.133, P=0.894)$, but we observed a weak positive correlation for PNC $(r=0.0417, t(3258)=2.379, P=0.0174)$.

We made the same different-ancestry GWAS comparisons for the EUR-ancestry individuals in the PNC and ABCD study populations (Figure 7). As was the case for AFR-ancestry individuals, we found no significant correlation between PGS computed from the PGC Freeze 2 EUR- and AFR-ancestry PTSD discovery GWAS. ${ }^{37}$ While we observed no significant correlation between the PGS computed using the PGC Freeze 1 EUR- and AFR-ancestry PTSD discovery GWAS for EUR-ancestry individuals in ABCD $(r=-0.00109, t(5813), P=0.934)$, we did observe a weak positive correlation for the EUR cohort of PNC $(r=0.0379, t(5237)=2.746, P=0.0065)$. 
We compared T2D PGS computed from an AFR-ancestry discovery GWAS ${ }^{40}$ to those computed using two EUR discovery GWAS ${ }^{38 ; 39}$ published by the Diabetes Genetics Replication and Meta-Analysis (DIAGRAM) consortium. The newer EUR-ancestry T2D discovery GWAS ${ }^{39}$ yielded PGS that were uncorrelated with those computed from the AFR-ancestry discovery GWAS $^{40}$ for the AFR-ancestry individuals in both PNC $(r=0.0185, t(3258)=1.055, P=0.292)$ and $\operatorname{ABCD}(r=0.0219, t(1739)=0.912, P=0.362)$. Similarly, there was no significant correlation between the different-ancestry T2D PGS that we computed for the EUR-ancestry individuals in $\operatorname{PNC}(r=0.0240, t(5237)=1.739, P=0.082)$ and $\operatorname{ABCD}(r=0.0224, t(5813)=1.71, P=0.0872)$. We observed a weak positive correlation between the PGS computed from the older EURancestry T2D discovery GWAS ${ }^{38}$ and the PGS computed from the AFR-ancestry T2D discovery $\mathrm{GWAS}^{40}$ for the PNC AFR cohort $(r=0.0432, t(3258)=2.469, P=0.0136)$, but there were no significant correlations between the two sets of PGS computed for the ABCD AFR cohort $(r=-$ $0.0458, t(1739)=-1.911, P=0.0562)$, the PNC EUR cohort $(r=0.00528, t(5237)=0.382, P=$ $0.703)$, or the ABCD EUR cohort $(r=0.0188, t(5813)=1.431, P=0.152)$.

We also computed different-ancestry PGS using EUR-and AFR-ancestry height discovery GWAS that we obtained from the Genetic Investigation of Anthropometric Traits (GIANT) consortium. ${ }^{41 ;} 42$ We observed significant positive correlations between the PGS computed from the newer EUR- and AFR-ancestry height discovery GWAS ${ }^{42}$ for the PNC AFR $(r=0.287$, $\left.t(3258)=17.09, P<2 \times 10^{-16}\right), \operatorname{ABCD} \operatorname{AFR}\left(r=0.306, t(1739)=13.42, P<2 \times 10^{-16}\right)$, PNC EUR $(r=$ $\left.0.403, t(5237)=31.82, P<2 \times 10^{-16}\right)$, and ABCD EUR $\left(r=0.404, t(5813)=33.69, P<2 \times 10^{-16}\right)$ cohorts. Likewise, we found significant positive correlations between the PGS computed from the older EUR-ancestry height discovery GWAS ${ }^{41}$ and the AFR-ancestry height discovery GWAS ${ }^{42}$ 
for the PNC AFR $\left(r=0.258, t(3258)=15.22, P<2 \times 10^{-16}\right)$, ABCD AFR $(r=0.312, t(1739)=13.68$, $\left.P<2 \times 10^{-16}\right), \operatorname{PNC} \operatorname{EUR}\left(r=0.335, t(5239)=25.25, P<2 \times 10^{-16}\right)$, and ABCD EUR $(r=0.327$, $\left.t(5813)=26.39, P<2 \times 10^{-16}\right)$ cohorts. As was the case for T2D, there was only one AFR-ancestry height discovery GWAS ${ }^{42}$ available to use for computing PGS.

The supplement includes complete statistical results for the comparisons between PGS computed from different discovery GWAS for the PNC AFR (Table S6), ABCD AFR (Table S7), PNC EUR (Table S8), and ABCD EUR (Table S9) cohorts.

\section{Quantile-Based Comparisons}

Given that there was little to no correlation between PGS computed from different discovery GWAS, we considered the possibility that there would be more stability if we focused on the individuals who had PGS located in the upper tail of the distribution, as those are the individuals who would presumably be most at risk for a disease trait. Considering the top $20 \%$, $10 \%$, and $5 \%$ of PC-adjusted standardized PGS, we counted how many individuals were jointly identified as being at or above a given percentile of the PGS computed from two different discovery GWAS. See the supplement for complete results of these analyses for the PNC AFR (Table S10), ABCD AFR (Table S11), PNC EUR (Table S12), and ABCD EUR (Table S13) cohorts.

As a baseline comparison, we determined the degree of overlap between the individuals in the top quantiles of PGS computed from two PRS-CS runs using the Freeze 2 AFR- and EURancestry PTSD discovery GWAS ${ }^{37}$ for the AFR (Figure 8A) and EUR (Figure 9A) PNC cohorts, respectively. Of the $n=3260$ individuals in the PNC AFR cohort, there are $n=652$ individuals with PGS at or above the $80^{\text {th }}$ percentile, $n=326$ with PGS at or above the $90^{\text {th }}$ percentile, and $n$ $=163$ with PGS at or above the $95^{\text {th }}$ percentile of PGS. We found an overlap of 644 of the 652 
AFR-ancestry individuals who had PGS at or above the $80^{\text {th }}$ percentile from the two runs using the same AFR-ancestry PTSD discovery GWAS, which is a $98.7 \%$ overlap. Comparable degrees of overlap were observed between the PNC AFR-ancestry individuals with PTSD PGS at or above the $90^{\text {th }}(318 / 326=0.975)$ and $95^{\text {th }}(161 / 163=0.988)$ percentiles. Similarly, the proportional overlap between the PTSD PGS computed from two PRS-CS runs using the Freeze 2 EURancestry PTSD discovery GWAS ${ }^{37}$ for the EUR-ancestry cohort ( $n=5239$ ) was $1026 / 1048=0.979$ at or above the $80^{\text {th }}$ percentile, $513 / 524=0.979$ at or above the $90^{\text {th }}$ percentile, and $255 / 262=$ 0.973 at or above the $95^{\text {th }}$ percentile.

The proportional overlap decreases if we consider PGS computed from two different same-ancestry discovery GWAS. For the PNC AFR-ancestry cohort (Figure 8B), adjusted standardized PGS computed from the Freeze $1^{36}$ and Freeze $2^{37}$ PTSD AFR-ancestry discovery GWAS (Figure $8 \mathrm{~B}$ ) had $53.6 \%$ of individuals in common at or above the $80^{\text {th }}$ percentile, $47.5 \%$ at or above the $90^{\text {th }}$ percentile, and $36.3 \%$ at or above the $95^{\text {th }}$ percentile. The decrease in proportional overlap was even more pronounced for PGS computed from two different EURancestry GWAS for the PNC EUR-ancestry cohort (Figure 9B). The proportion of overlap became progressively smaller as we considered progressively higher percentiles for PTSD, T2D, and height. Moreover, the amount of overlap was greatest for height and smallest for PTSD at each of the percentiles that we considered.

Proportional overlap was even more dramatically decreased when we compared the top quantiles of the PGS that had been computed from an AFR-ancestry discovery GWAS to those that had been computed from a EUR-ancestry discovery GWAS (Figures 8C and 9C). Note that Figures 8 and 9 only include comparisons for PNC between the PGS computed using the newer 
discovery GWAS if there was more than one comparison possible; full results are presented in the supplement (Tables S10-S13). For the AFR cohort of PNC, the proportional overlap ranged from a low of $4.91 \%$ for different-ancestry PTSD PGS at the $95^{\text {th }}$ percentile to a high of $32.8 \%$ for different-ancestry height PGS at the $80^{\text {th }}$ percentile, whereas the proportional overlap for the PNC EUR cohort ranged from 3.82\% for different-ancestry T2D PGS at the $95^{\text {th }}$ percentile to 38.1\% for height PGS at the $80^{\text {th }}$ percentile. For both the EUR and AFR cohorts, the general pattern is that proportional overlap is largest for different-ancestry PGS at the $80^{\text {th }}$ percentile and smallest at the $95^{\text {th }}$ percentile. Within a given percentile, the proportional overlap is largest for height and smallest for either PTSD or T2D.

\section{SNP Heritability}

We hypothesized that PGS would be more stable for traits with discovery GWAS that explain more of the genomic variance. As such, we used LDSC to compute the SNP heritability for each of the EUR-ancestry discovery GWAS that we used to compute PGS (Figure 10). The genetic correlation calculated by LDSC for each pair of GWAS essentially perfect, with the lowest $r_{g}=0.9225 \pm 0.1807$ for PTSD. We found that SNP heritability on the observed scale was highest for height ${ }^{42}\left(h_{S N P}^{2}=0.697 \pm 0.067\right)$ and lowest for $\operatorname{PTSD}^{37}\left(h_{S N P}^{2}=0.017 \pm 0.003\right)$. On the liability scale (which is only relevant for the two disease traits), SNP heritability was substantially higher for T2D than for PTSD (Table S14). On both the observed and liability scales, there was a clear difference between the SNP heritability of the two discovery GWAS that were used to compute PGS for each trait. For height and T2D, the SNPs included in the newer GWAS explained more phenotypic variance than did those in the older GWAS, but the opposite was true for PTSD. 


\section{Discussion}

We have demonstrated that the PGS computed from different discovery GWAS have little to no correlation at the level of the individual patient. While the correlation is better for highly heritable anthropometric traits (e.g., height), the lack of correlation for medical and psychiatric disorders like T2D and PTSD underscores the need to proceed cautiously with integrating PGS into precision medicine applications.

This lack of correlation is especially noteworthy given that it was observed for PGS computed using meta-GWAS that were produced by the PGC, ${ }^{36 ; 37}$ DIAGRAM, ${ }^{38 ;}{ }^{39}$ and GIANT ${ }^{39}$; ${ }^{41}$ consortia. The fact that even same-ancestry meta-GWAS computed by the same consortia using overlapping samples and SNPs (Table 1) would yield PGS that lack meaningful correlation at the individual level raises serious concerns. If PGS are going to be used clinically, then they need to be reproducible. Furthermore, judging PGS quality based primarily on population-level analytics, such as the area under the receiver operator characteristic curve (AUC), that depend on known case-control status will not be an effective approach for judging the validity of PGS computed for individual patients who do not necessarily have known phenotypes. ${ }^{35 ; 56}$

Even if stand-alone PGS are not yet useful clinically, they could still be used to help identify those patients at highest disease risk. ${ }^{57}$ For instance, PGS for psychiatric traits could be used in conjunction with environmental factors to identify adolescents most at risk for developing psychosis and other mental health disorders. ${ }^{16}$ We are actively pursuing such applications with the PNC and ABCD cohorts and have found that ancestry-specific PTSD PGS do indeed add predictive value to models that include other non-genetic factors. ${ }^{58}$ Nonetheless, 
we caution that it is dangerous to rely solely on PGS quantiles to identify at-risk patients.

Different discovery GWAS yielded PGS that did not identify the same patients at the top quantiles of the distribution, and the amount of overlap decreased as higher quantiles were considered (Figures 8 and 9; Tables S10-S13). Hence, the instinctive decision to focus only on the upper tail of the PGS distribution will not mitigate the lack of PGS stability across different discovery GWAS.

We chose to use the Bayesian PRS-CS Python package to compute PGS for this study. It has been demonstrated ${ }^{4}$ that Bayesian methods generally yield more predictive PGS than those produced via traditional $P$-value thresholding approaches. The advantage of PRS-CS over other Bayesian methods is that it employs a very robust Strawderman-Berger continuous shrinkage prior rather than a discrete mixture prior, which allows for more accurate multivariate modeling of local LD in the polygenic prediction. ${ }^{3}$ When enough MCMC iterations are used to ensure convergence of the underlying Gibbs sampler algorithm, PRS-CS yields very consistent posterior effects (Figure 2). PGS computed using the same discovery GWAS are highly correlated when computed using multiple PRS-CS runs (Figure 3), and others have previously shown that PGS computed from the same discovery GWAS are strongly correlated when computed using PRS-CS and other Bayesian and non-Bayesian approaches. ${ }^{4}$ Hence, the lack of PGS stability across discovery GWAS that we report here cannot be attributed to the stochastic nature of Bayesian methods; there must be differences between the discovery GWAS.

By choosing to use multiple generations of GWAS produced by the same consortia, we hoped to minimize potential methodological differences between the same-trait meta-GWAS. As expected, the genetic correlation between each pair of same-trait GWAS was nearly perfect, 
no doubt due to the large overlap between SNPs and samples within each pair (Table 1).

Initially, we had assumed that the newer GWAS in each pair would be the "better" GWAS since we thought that the larger sample size would yield more explanatory power. We cannot rule out this possibility for the T2D and height GWAS; in both cases, the newer EUR GWAS had higher SNP heritability than the older one (Figure 10). However, given that the opposite was true for the two PTSD GWAS, we have come to believe that bigger GWAS are not necessarily better.

Phenotyping quality should not be overlooked when choosing a discovery GWAS. Consortia have developed QC pipelines, such as RICOPILI, ${ }^{59}$ to harmonize genomic data from multiple cohorts prior to meta-analyzing. However, ensuring consistent phenotyping poses a continuing challenge, especially for case-control meta-GWAS analyses of non-continuous psychiatric traits like PTSD. The PGC Freeze 2 EUR-ancestry PTSD GWAS ${ }^{37}$ had a large increase in sample size due to the addition of samples from the UK Biobank, yet the SNP heritability went down compared to the Freeze 1 GWAS. ${ }^{36}$ One explanation could be that the UK Biobank PTSD phenotypes were derived from self-reported questionnaires, which are less reliable than the ascertainment procedures used by the smaller studies that comprised the original metaanalysis. ${ }^{60}$

It is not surprising that the two height GWAS had higher SNP heritability than the PTSD and T2D GWAS (Figure 10; Table S14). Height is an easily measured quantitative trait that is less susceptible to ascertainment bias than qualitative disease traits. Furthermore, environmental factors make substantial contributions to the development of both PTSD ${ }^{61}$ and T2D. ${ }^{62}$ Even so, LDSC gave an unusually high estimate of SNP heritability $\left(h_{S N P}^{2}=0.6967 \pm\right.$ 
0.0674 ) for the newer height GWAS. ${ }^{42}$ While it is possible that the LDSC calculations could have been biased due to the small number of SNPs, we believe that a plausible explanation lies in the design of this GWAS. Specifically, the newer height GWAS included a small number of targeted rare and low-frequency SNPs (MAF between $0.1 \%$ and $4.8 \%$ ) on a specially designed exome array rather than casting the same wide net as the earlier GWAS. This modification coupled with a substantially increased sample size and an easily ascertained quantitative trait could have yielded this improvement in explanatory power. It is tantalizing to speculate that a new class of genomic association studies that incorporate a smaller number of low-frequency and rare variants with large effects could improve our understanding of the genetic basis of complex traits beyond what can be inferred from large-scale GWAS alone. ${ }^{63}$ Such studies will be facilitated by the recent release of the Trans-Omics for Precision Medicine (TOPMed) ${ }^{64}$ Imputation Server, which should yield improved imputation accuracy for low-frequency and rare variants. Given that SNP heritability for psychiatric disorders generally underestimates the heritability calculated from family studies, ${ }^{65}$ it could be worthwhile to adopt this type of targeted approach coupled with more closely harmonized phenotyping for future genomic association studies of PTSD and other neuropsychiatric traits.

The previous heritability discussion is limited to the EUR-ancestry discovery GWAS. We chose not to use LDSC to compute SNP heritability for the AFR-ancestry GWAS due to evidence that it yields biased estimates for admixed populations. ${ }^{55}$ Given the relatively smaller sample sizes of the AFR GWAS (Table 1), you might expect that they explained less trait variance than the corresponding EUR GWAS. However, this was not necessarily the case. Using individuallevel data, the Freeze 2 PTSD GWAS authors ${ }^{37}$ demonstrated that SNP heritability was 
comparable for EUR- and AFR-ancestry males on the liability scale, and it was higher for AFRancestry females than for EUR-ancestry females and both groups of males. Likewise, the relatively stronger correlation between pairs of same-ancestry AFR PTSD PGS (Figure 4) and the higher degree of overlap between individuals at upper end of the PGS distribution (Figure 8B; Tables S10 and S11) as compared to same-ancestry EUR PTSD PGS (Figures 5 and 9B; Tables S12 and S13) is consistent with the possibility that the smaller AFR-ancestry PTSD GWAS (neither of which included UK Biobank samples) explained more variance than the EUR-ancestry GWAS did. Ideally, future work will be directed towards developing methodology to calculate unbiased estimates of SNP heritability for AFR-ancestry and other admixed populations from GWAS summary statistics. Popcorn, ${ }^{66}$ a Python package for calculating trans-ethnic genetic correlation from GWAS summary statistics, provides a preliminary step in this direction, as does cov$\operatorname{LDSC}^{55}$

Our results add to the growing body of evidence that PGS should be computed from an ancestrally matched discovery GWAS. It is well established that EUR-ancestry GWAS typically yield PGS that are less predictive for AFR and other non-EUR ancestry groups. $19 ; 21 ; 25 ; 30 ; 31 ; 34 ; 67-71$ We have further demonstrated that PGS computed from same-ancestry GWAS for PTSD and T2D are uncorrelated with those computed from different-ancestry GWAS for both AFR- and EUR-ancestry study participants (Figures 6 and 7), and we also found that there is very little overlap between the individuals in the upper tails of the PGS distributions computed using EURancestry GWAS as compared to those computed using AFR-ancestry GWAS (Figures 8c and 9c; Tables S10-S13). Given the dearth of AFR-ancestry and other non-EUR-ancestry discovery 
GWAS, our results underscore the urgent need for more high-powered GWAS analyses to be run for non-EUR ancestry populations.

We chose to study PTSD, T2D, and height because all three traits had publicly available GWAS for both EUR- and AFR-ancestry populations. Of these three, PTSD was the only trait that had two AFR-ancestry GWAS available for comparison purposes. While we focused our current work on the EUR- and AFR-ancestry individuals in the PNC and ABCD cohorts, we hope that methodology and GWAS data will soon exist to make it possible expand our analyses to the admixed American (AMR) and other ancestral groups that are also included in these cohorts (Figure 1). The recent release of PRS-CSx ${ }^{72}$ will make it possible to use discovery GWAS that include a combination of EAS-, AFR-, and EUR-ancestry samples. Although it offers an improvement over the current requirement that the discovery GWAS be limited to only one of these three ancestry groups, PRS-CSx still does not enable analyses of admixed samples from other genetic backgrounds.

Ultimately, we envision a future where genetic ancestry will not be a necessary consideration before computing PGS. Given that genetic ancestry is continuous, it is rather artificial to assign samples to discrete ancestry groups. ${ }^{26}$ Within the AFR-ancestry group alone, there is an enormous degree of genetic diversity. ${ }^{31 ; 73}$ We controlled for such diversity by calculating PGS separately for each ancestry group and then regressing out within-ancestry principal components from the standardized PGS. We are optimistic that new methods that incorporate local ancestry $33 ; 74$ will eventually allow us to embrace this diversity and compute stable, accurate PGS for admixed populations. Increasingly economical whole-genome sequencing ${ }^{75}$ coupled with expanded (i.e., less Eurocentric) genotyping arrays ${ }^{71}$ and improved 
imputation to diverse reference panels from TOPMed ${ }^{64}$ should also facilitate the further development of inclusive approaches, such as BOLT-LMM, ${ }^{76 ; 77}$ trans-ethnic GWAS, ${ }^{78}$ and multiethnic PGS. ${ }^{32}$ While it certainly would be easier to continue to focus PGS development on EURancestry populations, we do so at the grave risk of further exacerbating the inequities in medical care between EUR-ancestry populations and the rest of the world. ${ }^{29 ;} 79$

\section{Description of Supplemental Data}

Supplemental data include six figures and fourteen tables.

\section{Declaration of Interests}

RB reports serving on the scientific board and owning stock in Taliaz Health, with no conflict of interest relevant to this work. The other authors declare no competing interests.

\section{Acknowledgments}

This work was supported by the following National Institute of Mental Health grants:

5U01MH119690-02, 3U01MH119690-02, 5K23MH120437-02, and 5R01MH119219-02.

Data used in the preparation of this article were obtained from the Adolescent Brain Cognitive Development ${ }^{S M}(A B C D)$ Study (https://abcdstudy.org), held in the NIMH Data Archive (NDA). This is a multisite, longitudinal study designed to recruit more than 10,000 children age

9-10 and follow them over 10 years into early adulthood. The ABCD Study ${ }^{\circledR}$ is supported by the National Institutes of Health and additional federal partners under award numbers U01DA041048, U01DA050989, U01DA051016, U01DA041022, U01DA051018, U01DA051037, 
U01DA050987, U01DA041174, U01DA041106, U01DA041117, U01DA041028, U01DA041134,

U01DA050988, U01DA051039, U01DA041156, U01DA041025, U01DA041120, U01DA051038,

U01DA041148, U01DA041093, U01DA041089, U24DA041123, U24DA041147. A full list of

supporters is available at https://abcdstudy.org/federal-partners.html. A listing of participating

sites and a complete listing of the study investigators can be found

at https://abcdstudy.org/consortium members/. ABCD consortium investigators designed and implemented the study and/or provided data but did not necessarily participate in the analysis or writing of this report. This manuscript reflects the views of the authors and may not reflect the opinions or views of the NIH or ABCD consortium investigators.

Support for the collection of the data for Philadelphia Neurodevelopment Cohort (PNC) was provided by grant RC2MH089983 awarded to Raquel Gur and RC2MH089924 awarded to Hakon Hakonarson. Subjects were recruited and genotyped through the Center for Applied Genomics (CAG) at The Children's Hospital in Philadelphia (CHOP). Phenotypic data collection occurred at the CAG/CHOP and at the Brain Behavior Laboratory, University of Pennsylvania. 


\section{Web Resources}

McCarthy Group imputation checking perl script

(https://www.well.ox.ac.uk/ wrayner/tools/index.html\#Checking)

McCarthy Group genotyping chip strand and build files

(https://www.well.ox.ac.uk/ wrayner/strand/)

Plink 1.9

(https://www.cog-genomics.org/plink/1.9/)

Bcftools

(https://github.com/samtools/bcftools)

Michigan Imputation Server

(https://imputationserver.sph.umich.edu/index.html)

KING: Kinship-based Inference for GWAS

(http://people.virginia.edu/ wc9c/KING/index.html)

The R Project for Statistical Computing

(https://www.r-project.org)

R package 'e1071'

(https://cran.r-project.org/web/packages/e1071/e1071.pdf)

R package 'qqman'

(https://cran.r-project.org/web/packages/qqman/vignettes/qqman.html)

PRS-CS

(https://github.com/getian107/PRScs)

LDSC

(https://github.com/bulik/ldsc)

DIAGRAM GWAS summary statistics

(http://diagram-consortium.org/downloads.html)

GIANT GWAS summary statistics

(https://portals.broadinstitute.org/collaboration/giant/index.php/GIANT consortium data file

s)

Psychiatric Genomics Consortium GWAS summary statistics

(https://www.med.unc.edu/pgc/data-index/) 


\section{Data and Code Availability}

The PNC and $A B C D$ genomic datasets used in this study are available by application from dbGaP (phs00060) and NDAR (NDA \#2573), respectively. All discovery GWAS summary statistics and software used in this study are publicly available; see Web Resources for access information.

\section{References}

1. Vilhjálmsson, Bjarni J., Yang, J., Finucane, Hilary K., Gusev, A., Lindström, S., Ripke, S., Genovese, G., Loh, P.-R., Bhatia, G., Do, R., et al. (2015). Modeling Linkage Disequilibrium Increases Accuracy of Polygenic Risk Scores. The American Journal of Human Genetics 97, 576-592.

2. Lloyd-Jones, L.R., Zeng, J., Sidorenko, J., Yengo, L., Moser, G., Kemper, K.E., Wang, H., Zheng, Z., Magi, R., Esko, T., et al. (2019). Improved polygenic prediction by Bayesian multiple regression on summary statistics. Nature Communications 10, 5086.

3. Ge, T., Chen, C.-Y., Ni, Y., Feng, Y.-C.A., and Smoller, J.W. (2019). Polygenic prediction via Bayesian regression and continuous shrinkage priors. Nature Communications 10, 1776.

4. Ni, G., Zeng, J., Revez, J.A., Wang, Y., Ge, T., Restaudi, R., Kiewa, J., Nyholt, D.R., Coleman, J.R.I., Smoller, J.W., et al. (2020). A comprehensive evaluation of polygenic score methods across cohorts in psychiatric disorders. medRxiv, 2020.2009.2010.20192310.

5. Khera, A.V., Chaffin, M., Aragam, K.G., Haas, M.E., Roselli, C., Choi, S.H., Natarajan, P., Lander, E.S., Lubitz, S.A., Ellinor, P.T., et al. (2018). Genome-wide polygenic scores for common diseases identify individuals with risk equivalent to monogenic mutations. Nature Genetics 50, 1219-1224.

6. Gettler, K., Levantovsky, R., Moscati, A., Giri, M., Wu, Y., Hsu, N.-Y., Chuang, L.-S., Sazonovs, A., Venkateswaran, S., Korie, U., et al. (2021). Common and Rare Variant Prediction and Penetrance of IBD in a Large, Multi-ethnic, Health System-based Biobank Cohort. Gastroenterology 160, 1546-1557. 
7. Padilla-Martínez, F., Collin, F., Kwasniewski, M., and Kretowski, A. (2020). Systematic Review of Polygenic Risk Scores for Type 1 and Type 2 Diabetes. International Journal of Molecular Sciences 21.

8. Rao, A., and Knowles, J. (2019). Polygenic risk scores in coronary artery disease. Current Opinion in Cardiology 34, 435-440.

9. Dikilitas, O., Schaid, D.J., Kosel, M.L., Carroll, R.J., Chute, C.G., Denny, J.A., Fedotov, A., Feng, Q., Hakonarson, H., Jarvik, G.P., et al. (2020). Predictive Utility of Polygenic Risk Scores for Coronary Heart Disease in Three Major Racial and Ethnic Groups. The American Journal of Human Genetics 106, 707-716.

10. Graff, R.E., Cavazos, T.B., Thai, K.K., Kachuri, L., Rashkin, S.R., Hoffman, J.D., Alexeeff, S.E., Blatchins, M., Meyers, T.J., Leong, L., et al. (2021). Cross-cancer evaluation of polygenic risk scores for 16 cancer types in two large cohorts. Nature Communications 12, 970.

11. Zhou, X., Li, Y.Y.T., Fu, A.K.Y., and Ip, N.Y. (2021). Polygenic Score Models for Alzheimer's Disease: From Research to Clinical Applications. Frontiers in Neuroscience, NA.

12. Ronald, A., de Bode, N., and Polderman, T.J.C. (2021). Systematic Review: How the Attention-Deficit/Hyperactivity Disorder Polygenic Risk Score Adds to Our Understanding of ADHD and Associated Traits. Journal of the American Academy of Child \& Adolescent Psychiatry.

13. Mistry, S., Harrison, J.R., Smith, D.J., Escott-Price, V., and Zammit, S. (2018). The use of polygenic risk scores to identify phenotypes associated with genetic risk of bipolar disorder and depression: A systematic review. Journal of Affective Disorders 234, 148155.

14. Biederman, J., Green, A., DiSalvo, M., and Faraone, S.V. (2021). Can polygenic risk scores help identify pediatric bipolar spectrum and related disorders?: A systematic review. Psychiatry Research 299, 113843.

15. Mistry, S., Harrison, J.R., Smith, D.J., Escott-Price, V., and Zammit, S. (2018). The use of polygenic risk scores to identify phenotypes associated with genetic risk of schizophrenia: Systematic review. Schizophrenia Research 197, 2-8.

16. Murray, G.K., Lin, T., Austin, J., McGrath, J.J., Hickie, I.B., and Wray, N.R. (2021). Could Polygenic Risk Scores Be Useful in Psychiatry?: A Review. JAMA Psychiatry 78, 210-219. 
17. Lambert, S.A., Abraham, G., and Inouye, M. (2019). Towards clinical utility of polygenic risk scores. Human molecular genetics 28, R133-R142.

18. Torkamani, A., Wineinger, N.E., and Topol, E.J. (2018). The personal and clinical utility of polygenic risk scores. Nature Reviews Genetics 19, 581-590.

19. Wray, N.R., Lin, T., Austin, J., McGrath, J.J., Hickie, I.B., Murray, G.K., and Visscher, P.M. (2021). From Basic Science to Clinical Application of Polygenic Risk Scores: A Primer. JAMA Psychiatry 78, 101-109.

20. Wand, H., Lambert, S.A., Tamburro, C., Iacocca, M.A., O’Sullivan, J.W., Sillari, C., Kullo, I.J., Rowley, R., Dron, J.S., Brockman, D., et al. (2021). Improving reporting standards for polygenic scores in risk prediction studies. Nature 591, 211-219.

21. Peterson, R.E., Kuchenbaecker, K., Walters, R.K., Chen, C.-Y., Popejoy, A.B., Periyasamy, S., Lam, M., lyegbe, C., Strawbridge, R.J., Brick, L., et al. (2019). Genome-wide Association Studies in Ancestrally Diverse Populations: Opportunities, Methods, Pitfalls, and Recommendations. Cell 179, 589-603.

22. Choi, S.W., Mak, T.S.-H., and O'Reilly, P.F. (2020). Tutorial: a guide to performing polygenic risk score analyses. Nature Protocols 15, 2759-2772.

23. Wray, N.R., Yang, J., Hayes, B.J., Price, A.L., Goddard, M.E., and Visscher, P.M. (2013). Pitfalls of predicting complex traits from SNPs. Nature Reviews Genetics 14, 507-515.

24. Sirugo, G., Williams, S.M., and Tishkoff, S.A. (2019). The Missing Diversity in Human Genetic Studies. Cell 177, 26-31.

25. Duncan, L., Shen, H., Gelaye, B., Meijsen, J., Ressler, K., Feldman, M., Peterson, R., and Domingue, B. (2019). Analysis of polygenic risk score usage and performance in diverse human populations. Nature Communications 10, 3328.

26. Manolio, T.A. (2019). Using the Data We Have: Improving Diversity in Genomic Research. The American Journal of Human Genetics 105, 233-236.

27. Mills, M.C., and Rahal, C. (2019). A scientometric review of genome-wide association studies. Communications Biology 2, 9. 
28. Hindorff, L.A., Bonham, V.L., Brody, L.C., Ginoza, M.E.C., Hutter, C.M., Manolio, T.A., and Green, E.D. (2018). Prioritizing diversity in human genomics research. Nature Reviews Genetics 19, 175-185.

29. Martin, A.R., Kanai, M., Kamatani, Y., Okada, Y., Neale, B.M., and Daly, M.J. (2019). Clinical use of current polygenic risk scores may exacerbate health disparities. Nature Genetics $51,584-591$.

30. Martin, A.R., Gignoux, C.R., Walters, R.K., Wojcik, G.L., Neale, B.M., Gravel, S., Daly, M.J., Bustamante, C.D., and Kenny, E.E. (2017). Human Demographic History Impacts Genetic Risk Prediction across Diverse Populations. The American Journal of Human Genetics 100, 635-649.

31. Majara, L., Kalungi, A., Koen, N., Zar, H., Stein, D.J., Kinyanda, E., Atkinson, E.G., and Martin, A.R. (2021). Low generalizability of polygenic scores in African populations due to genetic and environmental diversity. bioRxiv, 2021.2001.2012.426453.

32. Márquez-Luna, C., Loh, P.-R., South Asian Type 2 Diabetes, C., The, S.T.D.C., and Price, A.L. (2017). Multiethnic polygenic risk scores improve risk prediction in diverse populations. Genetic Epidemiology 41, 811-823.

33. Bitarello, B.D., and Mathieson, I. (2020). Polygenic Scores for Height in Admixed Populations. G3 Genes|Genomes|Genetics 10, 4027-4036.

34. Grinde, K.E., Qi, Q., Thornton, T.A., Liu, S., Shadyab, A.H., Chan, K.H.K., Reiner, A.P., and Sofer, T. (2019). Generalizing polygenic risk scores from Europeans to Hispanics/Latinos. Genetic Epidemiology 43, 50-62.

35. Wald, N.J., and Old, R. (2019). The illusion of polygenic disease risk prediction. Genetics in Medicine 21, 1705-1707.

36. Duncan, L.E., Ratanatharathorn, A., Aiello, A.E., Almli, L.M., Amstadter, A.B., Ashley-Koch, A.E., Baker, D.G., Beckham, J.C., Bierut, L.J., Bisson, J., et al. (2018). Largest GWAS of PTSD ( $N=20070)$ yields genetic overlap with schizophrenia and sex differences in heritability. Mol Psychiatry 23, 666-673.

37. Nievergelt, C.M., Maihofer, A.X., Klengel, T., Atkinson, E.G., Chen, C.-Y., Choi, K.W., Coleman, J.R.I., Dalvie, S., Duncan, L.E., Gelernter, J., et al. (2019). International metaanalysis of PTSD genome-wide association studies identifies sex- and ancestry-specific genetic risk loci. Nature Communications 10, 4558. 
38. Scott, R.A., Scott, L.J., Mägi, R., Marullo, L., Gaulton, K.J., Kaakinen, M., Pervjakova, N., Pers, T.H., Johnson, A.D., Eicher, J.D., et al. (2017). An Expanded Genome-Wide Association Study of Type 2 Diabetes in Europeans. Diabetes 66, 2888.

39. Mahajan, A., Wessel, J., Willems, S.M., Zhao, W., Robertson, N.R., Chu, A.Y., Gan, W., Kitajima, H., Taliun, D., Rayner, N.W., et al. (2018). Refining the accuracy of validated target identification through coding variant fine-mapping in type 2 diabetes. Nature Genetics 50, 559-571.

40. Chen, J., Sun, M., Adeyemo, A., Pirie, F., Carstensen, T., Pomilla, C., Doumatey, A.P., Chen, G., Young, E.H., Sandhu, M., et al. (2019). Genome-wide association study of type 2 diabetes in Africa. Diabetologia 62, 1204-1211.

41. Wood, A.R., Esko, T., Yang, J., Vedantam, S., Pers, T.H., Gustafsson, S., Chu, A.Y., Estrada, K., Luan, J.a., Kutalik, Z., et al. (2014). Defining the role of common variation in the genomic and biological architecture of adult human height. Nature Genetics 46, 1173-1186.

42. Marouli, E., Graff, M., Medina-Gomez, C., Lo, K.S., Wood, A.R., Kjaer, T.R., Fine, R.S., Lu, Y., Schurmann, C., Highland, H.M., et al. (2017). Rare and low-frequency coding variants alter human adult height. Nature 542, 186-190.

43. Calkins, M.E., Merikangas, K.R., Moore, T.M., Burstein, M., Behr, M.A., Satterthwaite, T.D., Ruparel, K., Wolf, D.H., Roalf, D.R., Mentch, F.D., et al. (2015). The Philadelphia Neurodevelopmental Cohort: constructing a deep phenotyping collaborative. Journal of child psychology and psychiatry and allied disciplines 56, 1356-1369.

44. Glessner, J.T., Reilly, M.P., Kim, C.E., Takahashi, N., Albano, A., Hou, C., Bradfield, J.P., Zhang, H., Sleiman, P.M.A., Flory, J.H., et al. (2010). Strong synaptic transmission impact by copy number variations in schizophrenia. Proceedings of the National Academy of Sciences 107, 10584.

45. Uban, K.A., Horton, M.K., Jacobus, J., Heyser, C., Thompson, W.K., Tapert, S.F., Madden, P.A.F., and Sowell, E.R. (2018). Biospecimens and the ABCD study: Rationale, methods of collection, measurement and early data. Developmental Cognitive Neuroscience 32, 97106.

46. Chang, C.C., Chow, C.C., Tellier, L.C.A.M., Vattikuti, S., Purcell, S.M., and Lee, J.J. (2015). Second-generation PLINK: rising to the challenge of larger and richer datasets. GigaScience 4. 
47. Rayner, N.W. (2018). HRC-1000G-check-bim-v4.2.9. In.

(https://www.well.ox.ac.uk/ wrayner/tools/index.html\#Checking).

48. Das, S., Forer, L., Schönherr, S., Sidore, C., Locke, A.E., Kwong, A., Vrieze, S.I., Chew, E.Y., Levy, S., McGue, M., et al. (2016). Next-generation genotype imputation service and methods. Nature Genetics 48, 1284-1287.

49. Danecek, P., Bonfield, J.K., Liddle, J., Marshall, J., Ohan, V., Pollard, M.O., Whitewham, A., Keane, T., McCarthy, S.A., Davies, R.M., et al. (2020). Twelve years of SAMtools and BCFtools. arXiv 2012.10295.

50. Manichaikul, A., Mychaleckyj, J.C., Rich, S.S., Daly, K., Sale, M., and Chen, W.-M. (2010). Robust relationship inference in genome-wide association studies. Bioinformatics 26, 2867-2873.

51. Meyer, D., Dimitriadou, E., Hornik, K., Weingessel, A., Leisch, F., Chang, C.-C., and Lin, C.-C. (2020). Misc Functions of the Department of Statistics, Probability Theory Group (Formerly: E1071), TU Wien. In. (https://CRAN.R-project.org/package=e1071).

52. R Core Team. (2019). R: A language and environment for statistical computing.(Vienna, Austria: R Foundation for Statistical Computing).

53. Bulik-Sullivan, B.K., Loh, P.-R., Finucane, H.K., Ripke, S., Yang, J., Patterson, N., Daly, M.J., Price, A.L., Neale, B.M., and Schizophrenia Working Group of the Psychiatric Genomics, C. (2015). LD Score regression distinguishes confounding from polygenicity in genomewide association studies. Nature Genetics 47, 291-295.

54. Bulik-Sullivan, B., Finucane, H.K., Anttila, V., Gusev, A., Day, F.R., Loh, P.-R., Duncan, L., Perry, J.R.B., Patterson, N., Robinson, E.B., et al. (2015). An atlas of genetic correlations across human diseases and traits. Nature Genetics 47, 1236-1241.

55. Luo, Y., Li, X., Wang, X., Gazal, S., Mercader, J.M., Neale, B.M., Florez, J.C., Auton, A., Price, A.L., Finucane, H.K., et al. (2020). Estimating heritability and its enrichment in tissuespecific gene sets in admixed populations. bioRxiv, 503144.

56. Dudbridge, F. (2013). Power and Predictive Accuracy of Polygenic Risk Scores. PLOS Genetics 9, e1003348.

57. Gibson, G. (2019). On the utilization of polygenic risk scores for therapeutic targeting. PLOS Genetics 15, e1008060. 
58. Daskalakis, N.P., Schultz, L.M., Visoki, E., Moore, T.M., Argabright, S.T., Harnett, N.G., DiDomenico, G.E., Warrier, V., Almasy, L., and Barzilay, R. (2021). Contributions of PTSD polygenic risk and environmental stress to suicidality in preadolescents. medRxiv, 2021.2005.2030.21258082.

59. Lam, M., Awasthi, S., Watson, H.J., Goldstein, J., Panagiotaropoulou, G., Trubetskoy, V., Karlsson, R., Frei, O., Fan, C.C., De Witte, W., et al. (2020). RICOPILI: Rapid Imputation for COnsortias PIpeLIne. Bioinformatics 36, 930-933.

60. Xue, A., Jiang, L., Zhu, Z., Wray, N.R., Visscher, P.M., Zeng, J., and Yang, J. (2021). Genomewide analyses of behavioural traits are subject to bias by misreports and longitudinal changes. Nature Communications 12, 20211.

61. Wolf, E.J., Miller, M.W., Sullivan, D.R., Amstadter, A.B., Mitchell, K.S., Goldberg, J., and Magruder, K.M. (2018). A classical twin study of PTSD symptoms and resilience: Evidence for a single spectrum of vulnerability to traumatic stress. Depression and Anxiety 35, 132-139.

62. Dendup, T., Feng, X., Clingan, S., and Astell-Burt, T. (2018). Environmental Risk Factors for Developing Type 2 Diabetes Mellitus: A Systematic Review. Int J Environ Res Public Health 15, 78.

63. Crouch, D.J.M., and Bodmer, W.F. (2020). Polygenic inheritance, GWAS, polygenic risk scores, and the search for functional variants. Proceedings of the National Academy of Sciences 117, 18924.

64. Taliun, D., Harris, D.N., Kessler, M.D., Carlson, J., Szpiech, Z.A., Torres, R., Taliun, S.A.G., Corvelo, A., Gogarten, S.M., Kang, H.M., et al. (2021). Sequencing of 53,831 diverse genomes from the NHLBI TOPMed Program. Nature 590, 290-299.

65. Baselmans, B.M.L., Yengo, L., van Rheenen, W., and Wray, N.R. (2021). Risk in Relatives, Heritability, SNP-Based Heritability, and Genetic Correlations in Psychiatric Disorders: A Review. Biol Psychiatry 89, 11-19.

66. Brown, Brielin C., Ye, Chun J., Price, Alkes L., and Zaitlen, N. (2016). Transethnic GeneticCorrelation Estimates from Summary Statistics. The American Journal of Human Genetics 99, 76-88.

67. Curtis, D. (2018). Polygenic risk score for schizophrenia is more strongly associated with ancestry than with schizophrenia. Psychiatr Genet 28, 85-89. 
68. Reisberg, S., Iljasenko, T., Läll, K., Fischer, K., and Vilo, J. (2017). Comparing distributions of polygenic risk scores of type 2 diabetes and coronary heart disease within different populations. PLOS ONE 12, e0179238.

69. Wang, Y., Guo, J., Ni, G., Yang, J., Visscher, P.M., and Yengo, L. (2020). Theoretical and empirical quantification of the accuracy of polygenic scores in ancestry divergent populations. Nature Communications 11, 3865.

70. Gurdasani, D., Barroso, I., Zeggini, E., and Sandhu, M.S. (2019). Genomics of disease risk in globally diverse populations. Nature Reviews Genetics 20, 520-535.

71. Kim, M.S., Patel, K.P., Teng, A.K., Berens, A.J., and Lachance, J. (2018). Genetic disease risks can be misestimated across global populations. Genome Biology 19, 179.

72. Ruan, Y., Anne Feng, Y.-C., Chen, C.-Y., Lam, M., Sawa, A., Martin, A.R., Qin, S., Huang, H., and Ge, T. (2021). Improving Polygenic Prediction in Ancestrally Diverse Populations. medRxiv, 2020.2012.2027.20248738.

73. Pereira, L., Mutesa, L., Tindana, P., and Ramsay, M. (2021). African genetic diversity and adaptation inform a precision medicine agenda. Nature Reviews Genetics 22, 284-306.

74. Atkinson, E.G., Maihofer, A.X., Kanai, M., Martin, A.R., Karczewski, K.J., Santoro, M.L., Ulirsch, J.C., Kamatani, Y., Okada, Y., Finucane, H.K., et al. (2021). Tractor uses local ancestry to enable the inclusion of admixed individuals in GWAS and to boost power. Nature Genetics 53, 195-204.

75. Homburger, J.R., Neben, C.L., Mishne, G., Zhou, A.Y., Kathiresan, S., and Khera, A.V. (2019). Low coverage whole genome sequencing enables accurate assessment of common variants and calculation of genome-wide polygenic scores. Genome Medicine 11, 74.

76. Loh, P.-R., Kichaev, G., Gazal, S., Schoech, A.P., and Price, A.L. (2018). Mixed-model association for biobank-scale datasets. Nature Genetics 50, 906-908.

77. Loh, P.-R., Tucker, G., Bulik-Sullivan, B.K., Vilhjálmsson, B.J., Finucane, H.K., Salem, R.M., Chasman, D.I., Ridker, P.M., Neale, B.M., Berger, B., et al. (2015). Efficient Bayesian mixed-model analysis increases association power in large cohorts. Nature Genetics 47, 284-290.

78. Li, Y.R., and Keating, B.J. (2014). Trans-ethnic genome-wide association studies: advantages and challenges of mapping in diverse populations. Genome Medicine 6, 91. 
bioRxiv preprint doi: https://doi.org/10.1101/2021.06.18.449060; this version posted June 18, 2021. The copyright holder for this preprint (which was not certified by peer review) is the author/funder, who has granted bioRxiv a license to display the preprint in perpetuity. It is made available under aCC-BY-NC-ND 4.0 International license.

79. De La Vega, F.M., and Bustamante, C.D. (2018). Polygenic risk scores: a biased prediction? Genome Medicine 10, 100. 


\section{Figures}
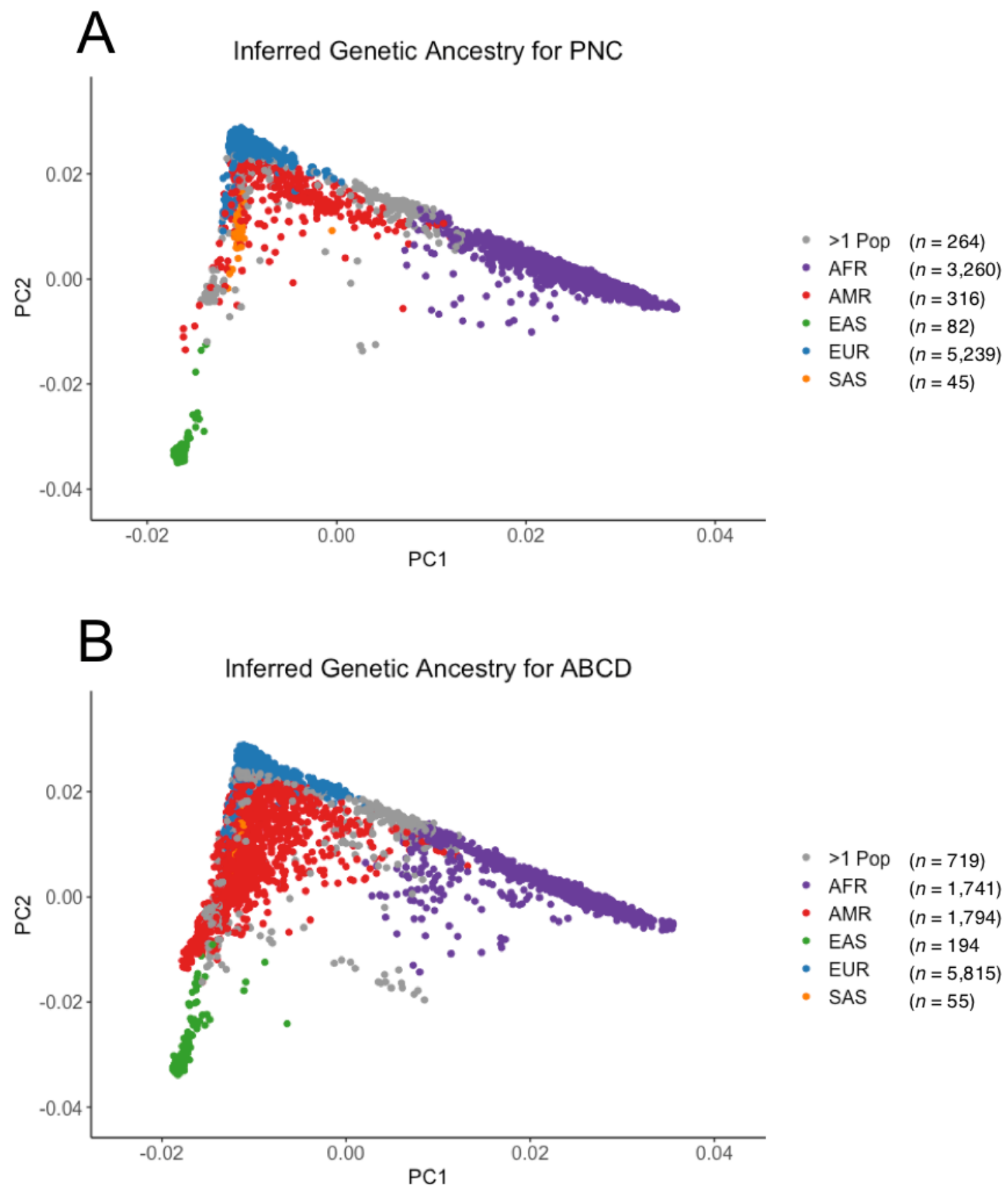

Figure 1. First and second principal components (PCs) of cohort genotypes. PCs were computed and projected to a 1000 Genomes reference using KING. ${ }^{50}$ Colors indicate inferred genetic ancestry for the (A) 9,206 Philadelphia Neurodevelopmental Cohort (PNC) and (B) 10,318 Adolescent Brain Cognitive Development ( $A B C D)$ genotyped samples. 

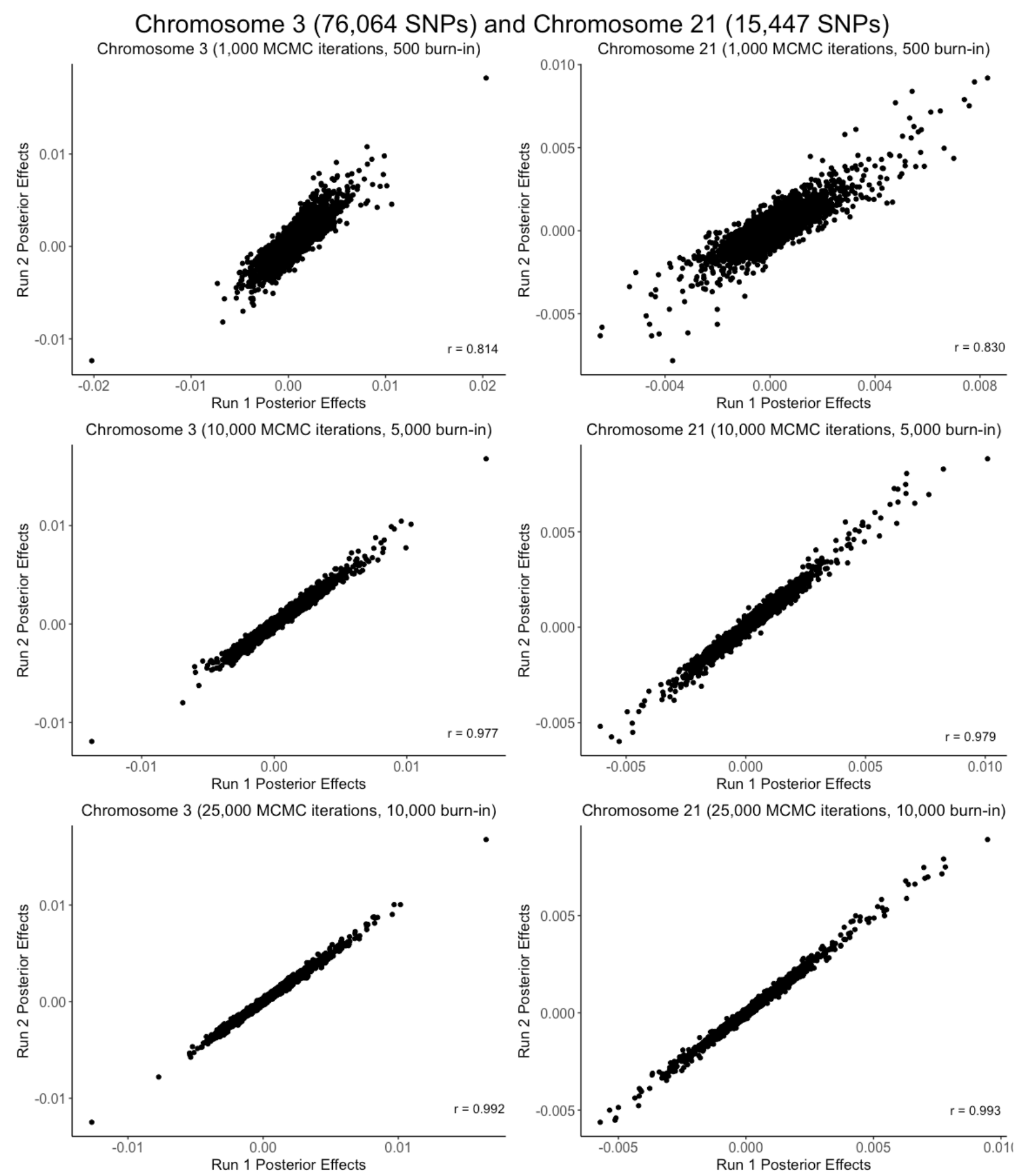

Figure 2. Reproducibility of Bayesian posterior effects computed by PRS-CS. As illustrated for chromosome 3 (76,064 SNPs) and chromosome 21 (15,447 SNPs) using the Nievergelt et al. $(2019)^{37}$ EUR PTSD discovery GWAS with the PNC EUR dataset, posterior effects were more strongly correlated between PRS-CS runs as the number of MCMC iterations (and burn-in iterations) increased. 

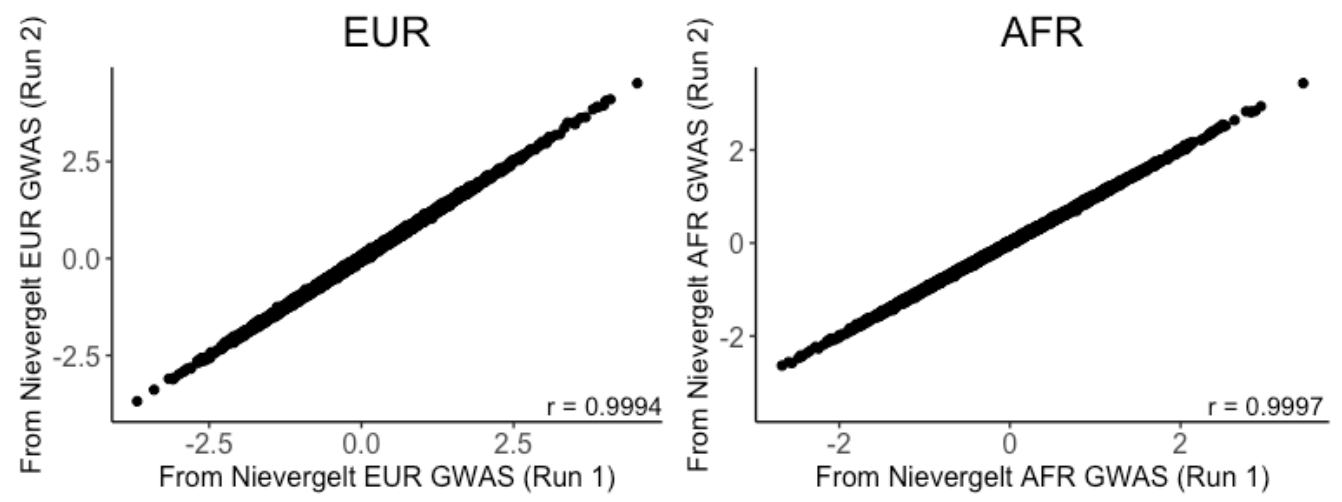

Figure 3. Reproducibility of PGS across multiple runs of PRS-CS. PC-adjusted standardized PGS computed from posterior effects generated by two runs of PRS-CS using the same PTSD discovery GWAS from Nievergelt et al. $(2019)^{37}$ had correlations greater than $r=0.999$ for both the EUR $(n=5239)$ and AFR $(n=3260)$ cohorts of PNC.
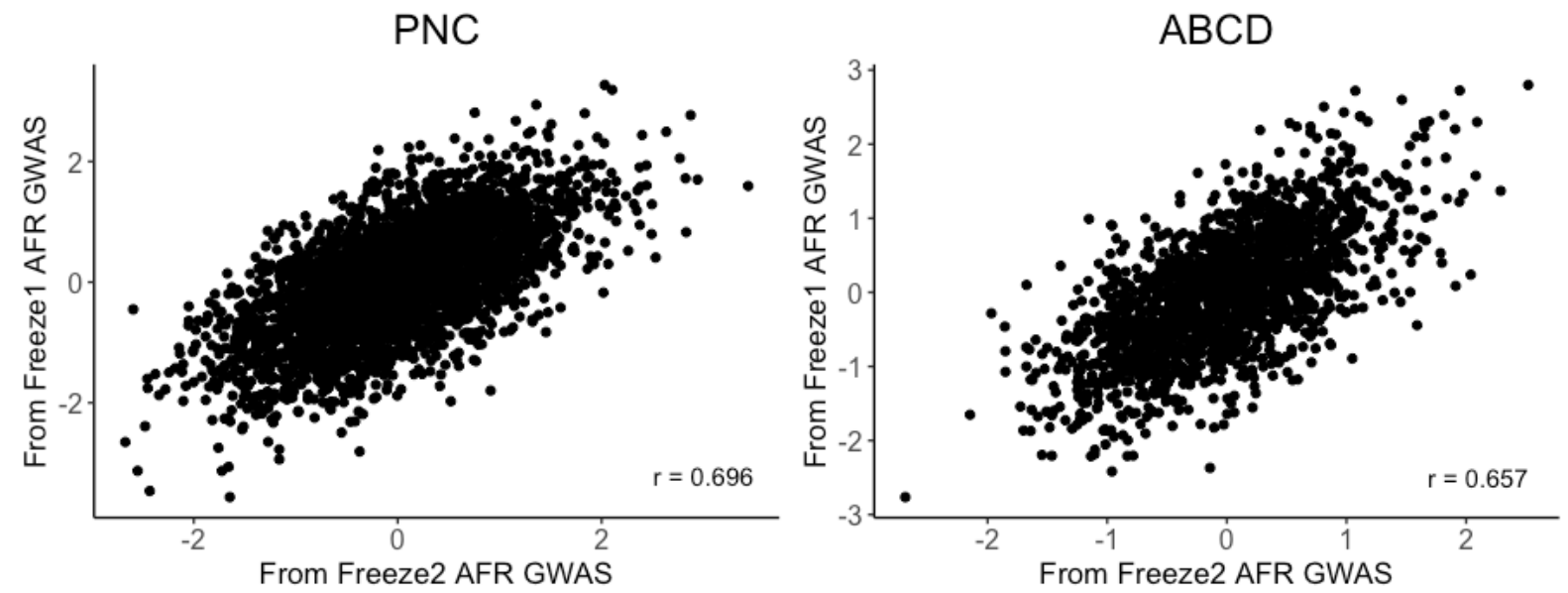

Figure 4. Correlation between PGS computed from two different AFR-ancestry PTSD discovery GWAS for AFR-ancestry individuals. Significant positive correlations were observed between the AFR PGS computed from the PGC Freeze $1^{36}$ and Freeze $2^{37}$ AFR PTSD GWAS for both the PNC $\left(r=0.696, t(3258)=55.26, P<2 \times 10^{-16}\right)$ and ABCD $(r=0.657, t(1739)=36.34, P<$ $\left.2 \times 10^{-16}\right)$ AFR cohorts. 

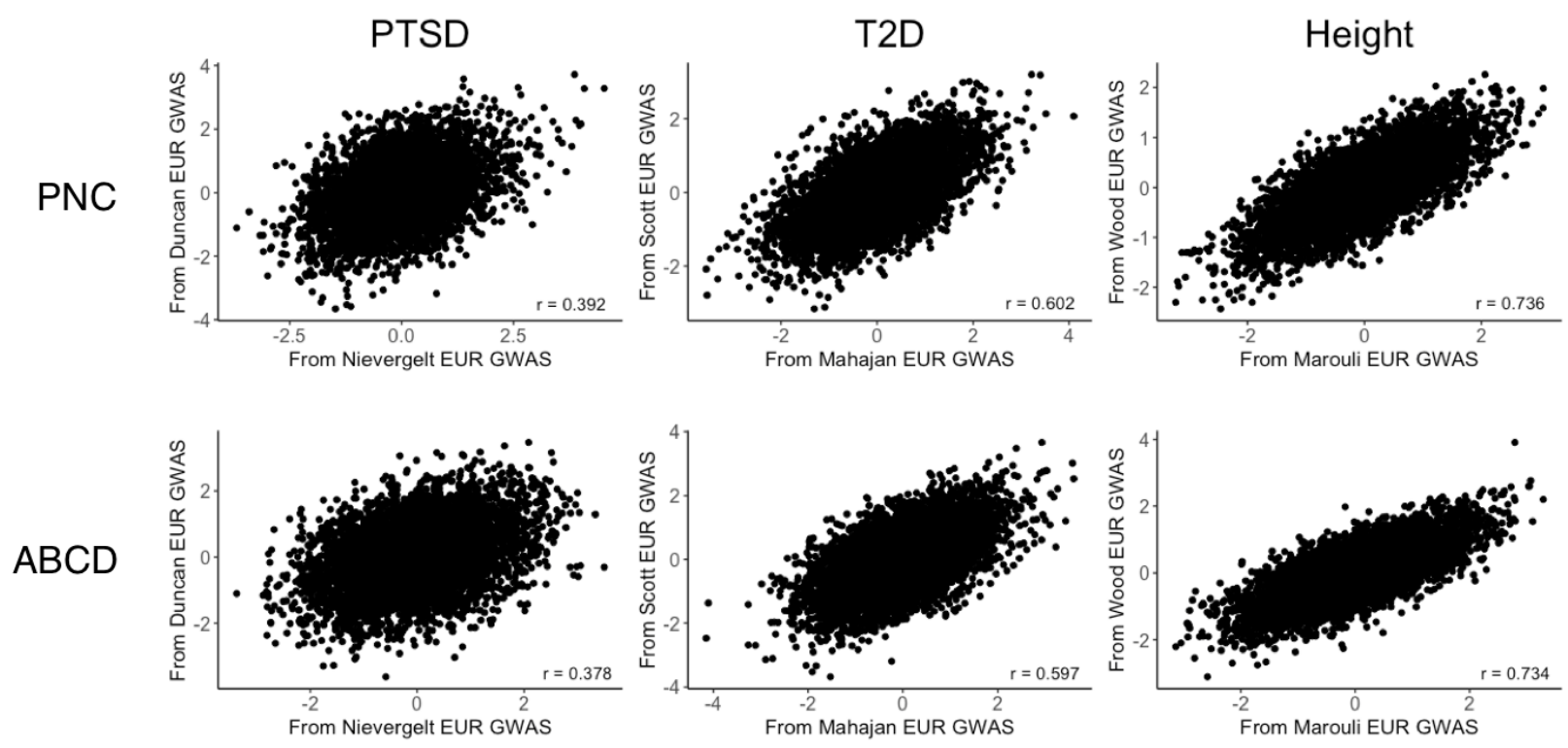

Figure 5. Correlation between PGS computed from two different EUR-ancestry discovery GWAS for EUR-ancestry individuals. Pairs of PGS computed for the EUR samples of PNC ( $n=$ 5239) and $A B C D(n=5815)$ using two different EUR discovery GWAS for PTSD, ${ }^{36 ; 37}{ }^{2} 2 D,{ }^{38 ;} 39$ and height $^{41 ; 42}$ all showed significant positive correlations.
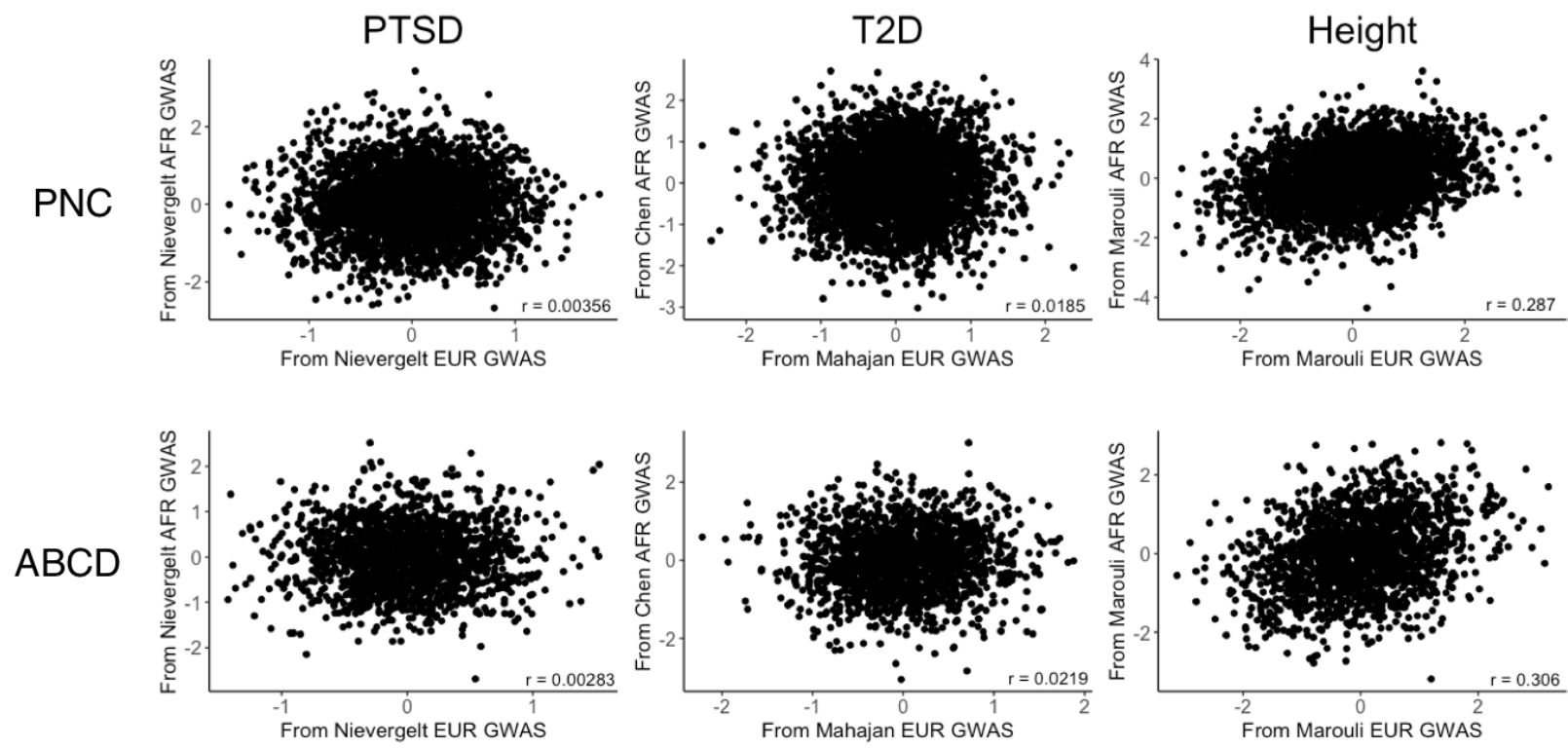

Figure 6. Correlation between PGS computed from AFR-ancestry and EUR-ancestry discovery GWAS for AFR-ancestry individuals. Pairs of PGS computed for the AFR samples of PNC and $A B C D$ from the newer EUR and AFR discovery GWAS were not significantly correlated for either $\mathrm{PTSD}^{36 ;} 37$ or $\mathrm{T} 2 \mathrm{D},{ }^{39 ;} 40$ but there was a significant positive correlation for height. ${ }^{42}$ 

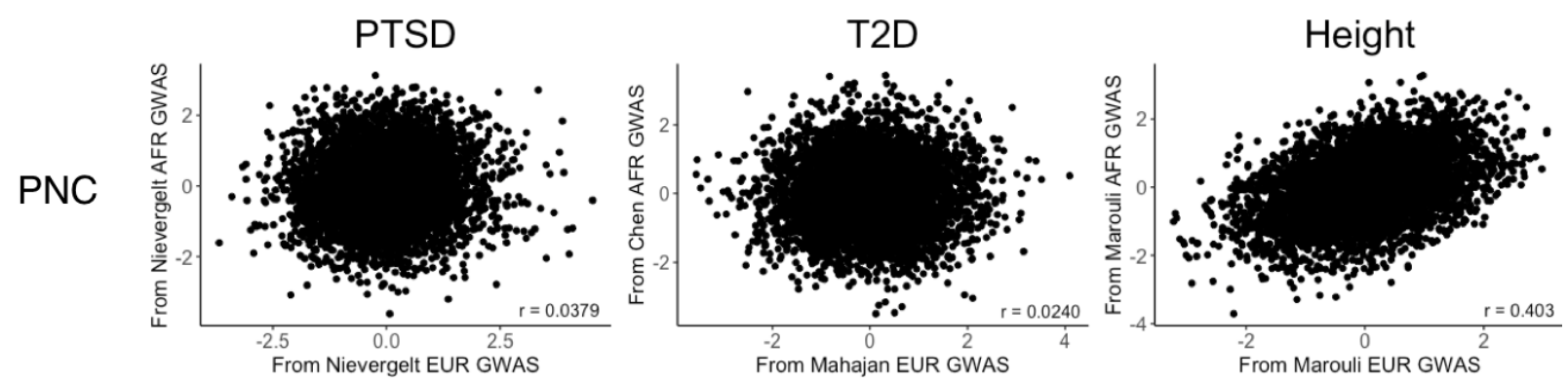

$\mathrm{ABCD}$
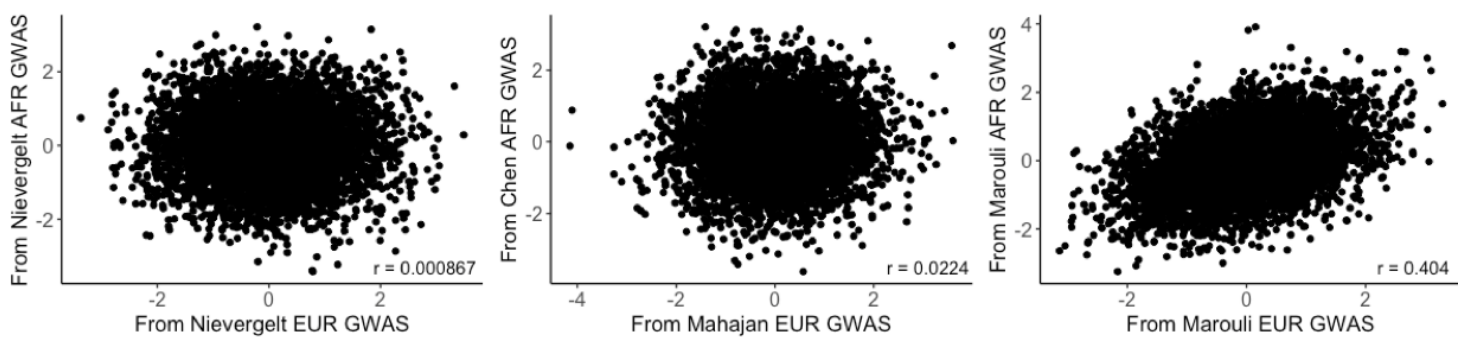

Figure 7. Correlation between PGS computed from EUR-ancestry and AFR-ancestry discovery GWAS for EUR-ancestry individuals. Pairs of PGS computed for the EUR samples of PNC and $A B C D$ from the newer EUR and AFR discovery GWAS were not significantly correlated for either $\mathrm{PTSD}^{36 ; 37}$ or T2D, ${ }^{39} ; 40$ but there was a significant positive correlation for height. ${ }^{42}$ 


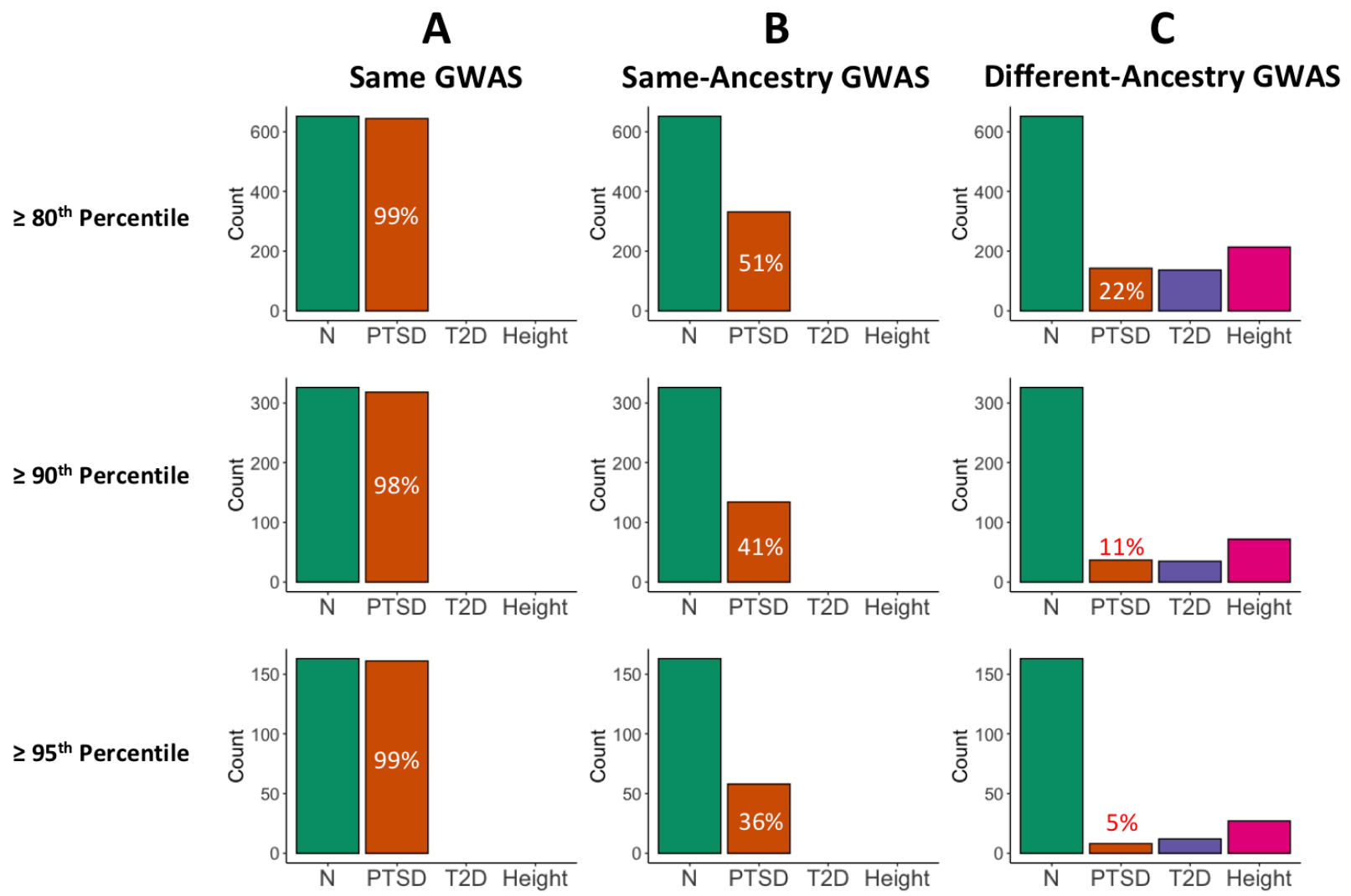

Figure 8. Comparison of the samples comprising the top PGS quantiles for the PNC AFR cohort. A. The samples located at the top $20 \%, 10 \%$, and $5 \%$ of the PTSD PGS distribution were virtually the same when PGS were computed twice using the same discovery GWAS. For example, 644 out of the 652 samples $(98.7 \%)$ at or above the $80^{\text {th }}$ percentile were the same between the two batches of PGS. B. The overlap between samples at all three quantiles dropped substantially when the PGS computed from the AFR PGC Freeze1 PTSD discovery GWAS $^{36}$ were compared to those computed from the AFR Freeze2 PTSD discovery GWAS, ${ }^{37}$ with the degree of overlap being reduced at higher quantiles. C. The degree of overlap was further reduced when comparing PGS computed from an AFR-ancestry discovery GWAS to those computed from an EUR-ancestry GWAS for PTSD, ${ }^{37} \mathrm{~T} 2 \mathrm{D},{ }^{39 ;} 40$ and height. ${ }^{42}$ For context, the green bars depict the number of samples included at or above the $80^{\text {th }}$ percentile $(n=652)$, $90^{\text {th }}$ percentile $(n=326)$, and $95^{\text {th }}$ percentile $(n=163)$. Full results can be found in Table S6. 


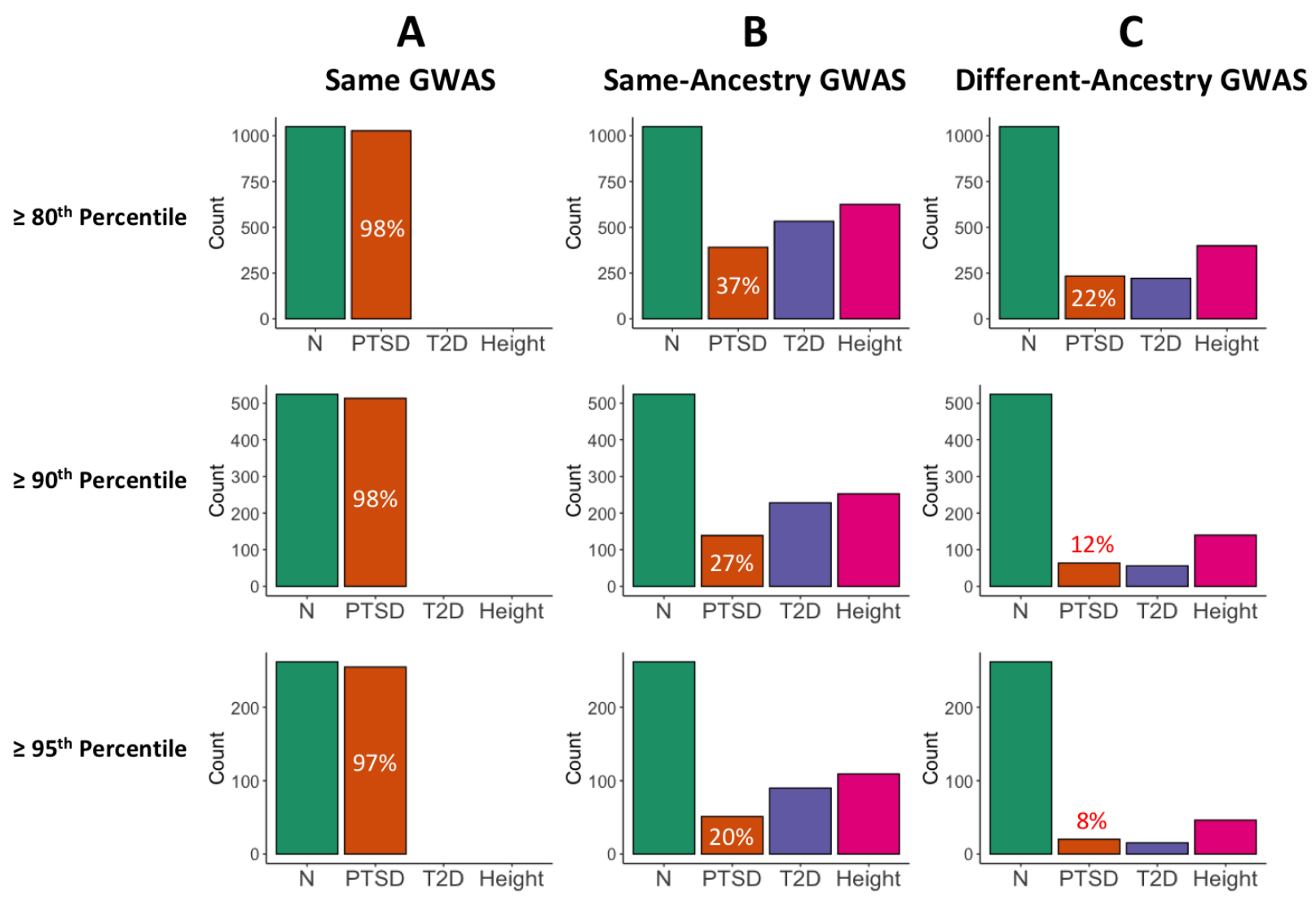

Figure 9. Comparison of the samples comprising the top PGS quantiles for the PNC EUR cohort. A. The EUR samples located within the top $20 \%, 10 \%$, and $5 \%$ of the PTSD PGS distribution were nearly the same when PGS were computed twice using the same EUR discovery GWAS. ${ }^{37}$ For example, 1026 out of the 1048 samples $(97.9 \%)$ at or above the $80^{\text {th }}$ percentile were the same between the two runs of PRS-CS. B. The overlap between samples at all three quantiles dropped substantially when the PGS computed from two different EUR discovery GWAS were compared for PTSD, ${ }^{36 ; 37} \mathrm{T2D},{ }^{38 ; 39}$ and height. ${ }^{41 ; 42}$ C. The degree of overlap was dramatically reduced when comparing PGS computed from an AFR-ancestry discovery GWAS to those computed from an EUR-ancestry GWAS for PTSD, ${ }^{37}$ T2D, ${ }^{39 ; 40}$ and height. ${ }^{41 ; 42}$ Green bars depict the number of samples included at or above the $80^{\text {th }}$ percentile $(n$ $=1048), 90^{\text {th }}$ percentile $(n=524)$, and $95^{\text {th }}$ percentile $(n=262)$. Full results can be found in Table S8. 


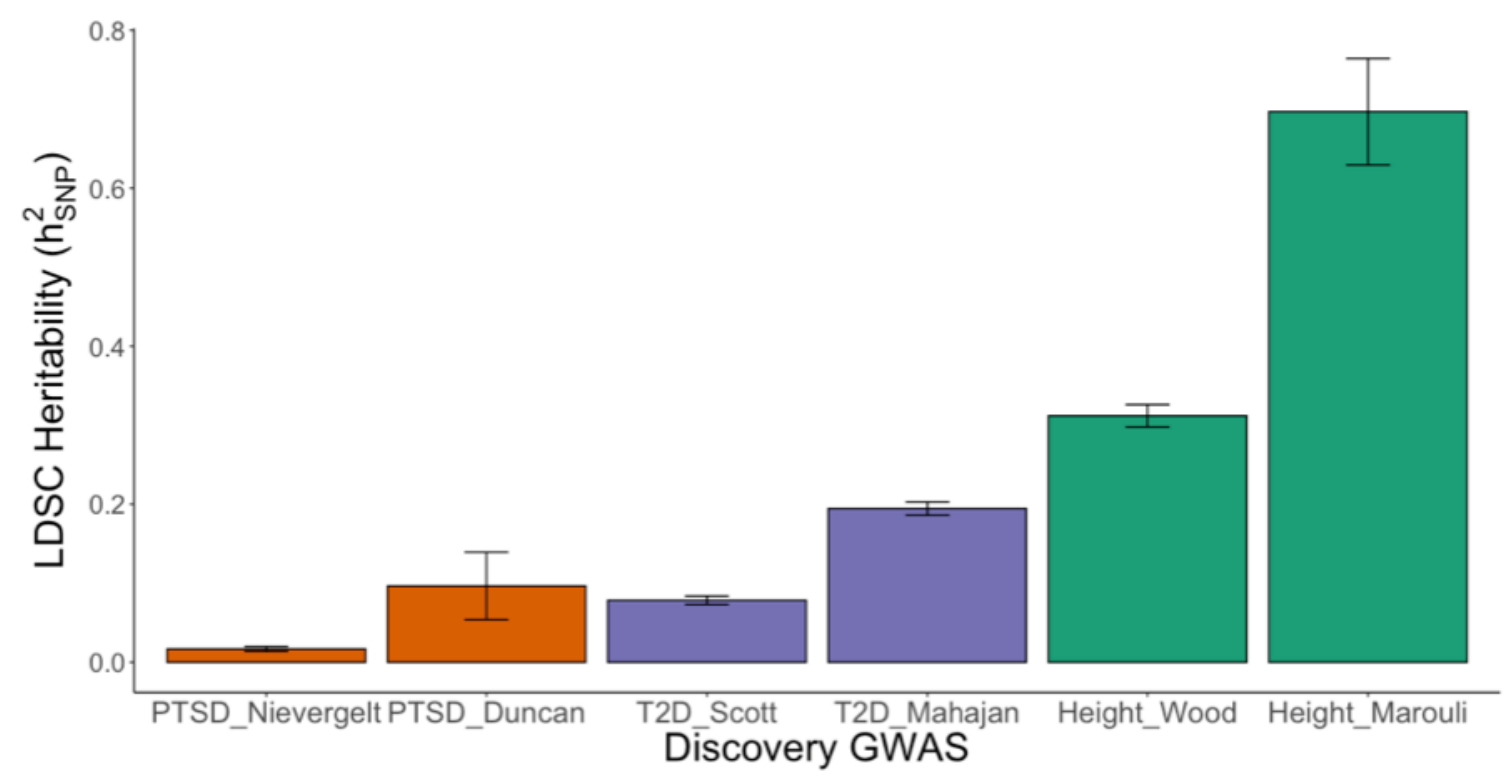

Figure 10. SNP heritability $\left(\boldsymbol{h}_{S N P}^{2}\right)$ observed-scale estimates calculated using LDSC for the European-ancestry height, PTSD, and T2D discovery GWAS that were used to compute PGS. Error bars denote standard error computed via a block-jackknife procedure. Heritability was highest for height, with the newer discovery GWAS by Marouli et al. (2017) ${ }^{42}$ showing greater heritability than the GWAS by Wood et al. (2014) ${ }^{41}$. The newer T2D discovery GWAS by Mahajan et al. (2018) ${ }^{39}$ likewise showed greater heritability than the older GWAS by Scott et al. (2017). ${ }^{38}$ PTSD showed the lowest heritability, with the older discovery GWAS by Duncan et al. $(2018)^{36}$ having greater heritability than the GWAS by Nievergelt et al. (2019). ${ }^{37}$ 


\section{Tables}

Table 1. Discovery GWAS used to compute polygenic scores with PRS-CS

\begin{tabular}{|c|c|c|c|c|c|}
\hline Trait & Discovery GWAS & $\begin{array}{l}\text { GWAS } \\
\text { Ancestry }\end{array}$ & $\begin{array}{c}\text { SNP Sample } \\
\text { Size }^{a} \\
\text { (for PRS-CS) }\end{array}$ & $\begin{array}{l}\text { SNP Count }{ }^{\mathrm{b}} \text { for } \\
\text { PNC PGS } \\
\text { Calculations }\end{array}$ & $\begin{array}{l}\text { SNP Count for } \\
\text { ABCD PGS } \\
\text { Calculations }\end{array}$ \\
\hline \multirow[t]{4}{*}{ PTSD } & \multirow{2}{*}{$\begin{array}{c}\text { Nievergelt et al. }(2019)^{37} \\
\text { (Freeze } 2 \text { PGC) }\end{array}$} & AFR & 11,321 & $1,162,502$ & $1,064,574$ \\
\hline & & EUR & 70,237 & $1,087,435$ & $1,016,161$ \\
\hline & \multirow{2}{*}{$\begin{array}{c}\text { Duncan et al. (2018) } \\
\text { (Freeze 1 PGC) }\end{array}$} & AFR & 9,691 & $1,157,302$ & $1,059,197$ \\
\hline & & EUR & 9,954 & $1,086,644$ & $1,015,369$ \\
\hline \multirow[t]{3}{*}{ T2D } & Chen et al. $(2019)^{40}$ & AFR & 4,146 & $1,114,936$ & $1,020,579$ \\
\hline & $\begin{array}{c}\text { Scott et al. }(2017)^{38} \\
\text { (DIAGRAM) }\end{array}$ & EUR & 152,599 & $1,087,724$ & $1,016,440$ \\
\hline & $\begin{array}{c}\text { Mahajan et al. (2018) } \\
\text { (DIAGRAM) }\end{array}$ & EUR & 231,420 & $1,089,613$ & $1,018,372$ \\
\hline \multirow[t]{3}{*}{ Height } & \multirow{2}{*}{$\begin{array}{c}\text { Marouli et al. }(2017)^{42} \\
\text { (GIANT) }\end{array}$} & AFR & 27,494 & 18,580 & 15,720 \\
\hline & & EUR & 381,625 & 18,035 & 15,767 \\
\hline & $\begin{array}{c}\text { Wood et al. }(2014)^{41} \\
\text { (GIANT) }\end{array}$ & EUR & 252,048 & 987,760 & 920,889 \\
\hline
\end{tabular}

PTSD, post-traumatic stress disorder; T2D, type 2 diabetes; PNC, Philadelphia

Neurodevelopmental Cohort; PGS, polygenic score; ABCD, Adolescent Brain Cognitive

Development Study; PGC, Psychiatric Genomics Consortium; DIAGRAM, Diabetes Genetics

Replication and Meta-Analysis Consortium; GIANT, Genetic Investigation of Anthropometric

Traits Consortium.

aPRS-CS requires a single SNP sample size; see Supplemental Methods for details.

'The "SNP count" is the number of SNPs in common between the discovery GWAS, the PRS-CS LD panel, and the genomic dataset. 